\title{
Conditional Deletion of Foxg1 Alleviates Demyelination and Facilitates Remyelination via the Wnt Signaling Pathway in Cuprizone-Induced Demyelinated Mice
}

\author{
Fuxing Dong ${ }^{1,2} \cdot$ Dajin Liu $^{1} \cdot$ Feiyu Jiang ${ }^{1}$ Yaping Liu ${ }^{1} \cdot$ Xiuxiang $\mathrm{Wu}^{1} \cdot$

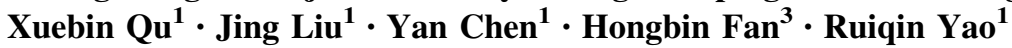

Received: 19 January 2020 / Accepted: 31 May 2020/Published online: 5 October 2020

(C) The Author(s) 2020

\begin{abstract}
The massive loss of oligodendrocytes caused by various pathological factors is a basic feature of many demyelinating diseases of the central nervous system (CNS). Based on a variety of studies, it is now well established that impairment of oligodendrocyte precursor cells (OPCs) to differentiate and remyelinate axons is a vital event in the failed treatment of demyelinating diseases. Recent evidence suggests that Foxgl is essential for the proliferation of certain precursors and inhibits premature neurogenesis during brain development. To date, very little attention has been paid to the role of Foxgl in the proliferation and differentiation of OPCs in demyelinating diseases of the CNS. Here, for the first time, we examined the effects of Foxgl on demyelination and remyelination in the brain using a cuprizone (CPZ)induced mouse model. In this work, 7-week-old Foxg1 conditional knockout and wild-type (WT) mice were fed a diet containing $0.2 \% \mathrm{CPZ}$ w/w for 5 weeks, after which $\mathrm{CPZ}$ was withdrawn to enable remyelination. Our results demonstrated that, compared with WT mice, Foxgl-
\end{abstract}

Fuxing Dong and Dajin Liu have contributed equally to this work.

Hongbin Fan

fandoc@163.com

$\triangle$ Ruiqin Yao

wenxi_yao@163.com

1 Department of Cell Biology and Neurobiology, Xuzhou Key Laboratory of Neurobiology, Jiangsu Key Laboratory of New Drug Research and Clinical Pharmacy, Xuzhou Medical University, Xuzhou 221004, China

2 Public Experimental Research Center, Xuzhou Medical University, Xuzhou 221004, China

3 Department of Neurology, Affiliated Hospital of Xuzhou Medical University, Xuzhou 221006, China knockout mice exhibited not only alleviated demyelination but also accelerated remyelination of the demyelinated corpus callosum. Furthermore, we found that Foxgl knockout decreased the proliferation of OPCs and accelerated their differentiation into mature oligodendrocytes both in vivo and in vitro. Wnt signaling plays a critical role in development and in a variety of diseases. GSK-3 $\beta$, a key regulatory kinase in the Wnt pathway, regulates the ability of $\beta$-catenin to enter nuclei, where it activates the expression of Wnt target genes. We then used SB216763, a selective inhibitor of GSK-3 $\beta$ activity, to further demonstrate the regulatory mechanism by which Foxgl affects OPCs in vitro. The results showed that SB216763 clearly inhibited the expression of GSK-3 $\beta$, which abolished the effect of the proliferation and differentiation of OPCs caused by the knockdown of Foxgl. These results suggest that Foxgl is involved in the proliferation and differentiation of OPCs through the Wnt signaling pathway. The present experimental results are some of the first to suggest that Foxgl is a new therapeutic target for the treatment of demyelinating diseases of the CNS.

Keywords Foxg1 - Oligodendrocyte precursor cells . Demyelination - Remyelination $\cdot$ Wnt

\section{Introduction}

In the central nervous system (CNS), oligodendrocytes (OLs) are myelin-forming glial cells that play vital roles in rapid impulse conduction and normal axonal functions. Current studies have demonstrated that inflammatory stimuli or immune attacks can damage the myelin sheath, leading to OL death and myelin sheath loss, such as that observed in multiple sclerosis (MS), the most common 
demyelinating disease [1]. It has been reported that in early MS lesions, neural stem cells (NSCs) rooted in the region of the lateral ventricle-subventricular zone migrate, proliferate, and differentiate into oligodendrocyte progenitor cells (OPCs) induced by the injury signal of the myelin sheath and later differentiate to myelinating OLs to repair the damaged myelin [2]. However, OPCs are slow-cycling cells, and only long-term nutritional factors can stimulate their rapid proliferation and differentiation [3]. Furthermore, the repair ability of endogenous remyelination is limited, resulting in difficulties in the regeneration/repair of the myelin sheath in demyelinating diseases. Therefore, elucidating the regulatory mechanism of the correct differentiation of OPCs is crucial for understanding the myelination and remyelination processes in the CNS and is also fundamental to the treatment of demyelinating diseases [4].

Foxg1, also referred to as brain factor-1, is an important member of the Forkhead box transcription factor family, which plays dual roles in transcriptional activation and transcriptional repression [5]. Foxgl is widely expressed in the cerebral cortex and is critical to the development of the CNS [6]. One or two homologous chromosome Foxgl gene translocations, deletions, or mutations are closely related to various diseases associated with the occurrence and development of the CNS [7, 8]. Previous studies have shown that loss of function or downregulation of Foxg1 reduces the proliferation of neural precursor cells, accompanied by early differentiation into neurons [9, 10]. In addition, Tian et al. found that Foxgl deletion increases the numbers of neurons and astrocytes at an early stage after birth in conditional Foxgl gene-knockout mice, and they speculated that Foxg1 might inhibit neurogenesis and glial cell formation in the early stage after birth [11]. However, the regulation of Foxgl in the development and differentiation of OPCs is still uncertain.

The Wnt signaling pathway plays an important role in the development and myelination of OPCs $[12,13]$. GSK$3 \beta$, a key kinase in the Wnt signaling pathway, negatively regulates the entry of catenin and then affects the expression of Wnt target genes [14]. Recent studies have demonstrated that GSK-3 $\beta$ activation promotes OPC differentiation, which may be mediated by the accumulation of nuclear $\beta$-catenin [15]. Nevertheless, whether the effect of Foxgl on the development and maturation of OPCs is associated with the Wnt signaling pathway has not been reported.

To investigate the role of Foxgl in the proliferation and differentiation of oligodendrocyte lineage cells and myelin regeneration, we conditionally knocked out Foxgl in vivo to assess the loss and repair of myelin during demyelinating injury in mice, and we further explored its regulatory mechanism in vitro. Our findings revealed a previously- unknown function of Foxgl in oligodendrocyte lineage cells, suggesting that downregulating the expression of Foxgl in the treatment of demyelinating CNS diseases may alleviate demyelination and promote remyelination, thus providing a new gene therapy target for demyelinating CNS diseases such as MS.

\section{Materials and Methods}

\section{Animals}

Foxgl cKO mice (1 male Nestin-CreER ${ }^{\mathrm{TM}}$; Foxgl $1^{f / f l}$ mouse; 2 female Foxg $1^{f / f l}$ mice) were donated by Professor Chunjie Zhao from the Medical School of Southeast University. Wild-type ICR mice were purchased from the Experimental Animal Center of Xuzhou Medical University. All mice were housed with free access to food and water under a 12/12-h dark/light cycle and specific pathogen-free conditions. For Foxgl conditional disruption in neural stem/progenitor cells (NSPCs), Nestin-CreER ${ }^{\mathrm{TM}}$ mice were crossed with Foxg $1^{f / f l}$ mice and induced with tamoxifen (TM; Sigma-Aldrich, St. Louis, MO, USA). The genotypes of all mice were determined by PCR analysis of tail genomic DNA with appropriate primers. Male and female mice were used for all experiments without bias. All experiments were performed according to the guidelines approved by Xuzhou Medical University Experimental Animal Ethics Committee.

The TM was dissolved in corn oil to a concentration of $10 \mathrm{mg} / \mathrm{mL}$ by shaking the solution for $3 \mathrm{~h}$ at $37^{\circ} \mathrm{C}$. For $\mathrm{CreER}^{\mathrm{TM}}$-mediated recombination, $\mathrm{TM}$ was intraperitoneally injected into mice at $1 \mathrm{mg} / 20 \mathrm{~g}$ body weight $[16,17] 3$ times on alternate days.

\section{Experimental Model}

Experimental demyelination was induced by feeding 7-week-old male mice $0.2 \%$ (w/w) cuprizone (CPZ, Sigma Aldrich, St. Louis, MO, USA) mixed into ground standard rodent chow $[18,19]$. The main ingredients in the normal diet for mice in this experiment were crude protein $(\geq 180$ $\mathrm{g} / \mathrm{kg})$, crude fat $(\geq 40 \mathrm{~g} / \mathrm{kg})$, and crude fiber $(\leq 50 \mathrm{~g} / \mathrm{kg})$. These mice were fed CPZ for 5 weeks and then allowed to remyelinate for 2 weeks with normal food.

\section{Experimental Groups}

Depending on the experimental purpose, mice were sacrificed at different times as follows: (1) to assess the knockout efficiency of Foxg1, 8 wild-type mice (WT group) and 8 Foxg 1 cKO mice (cKO group) were sacrificed on day 7 after TM injection; (2) at the end of 5 weeks of 
CPZ administration, 32 mice in 4 groups (WT, WT+CPZ, $\mathrm{cKO}$, and $\mathrm{cKO}+\mathrm{CPZ} ; 8$ mice per group) were sacrificed to explore the effects of Foxgl on CPZ-induced demyelination and OPC differentiation; (3) at the end of 4 weeks of CPZ administration, 24 mice in 3 groups (WT, WT $+\mathrm{CPZ}$ and $\mathrm{cKO}+\mathrm{CPZ} ; 8$ mice per group) were sacrificed to explore the effects of Foxgl on OPC proliferation; and (4) another 24 mice from the same groups ( 8 mice per group) were sacrificed to investigate the effects of Foxgl on remyelination after 5 weeks of $\mathrm{CPZ}$ administration and another 2 weeks of recovery with normal chow. All mice received the same amount of chow each day.

In the OPC proliferation study, the mice were intraperitoneally injected with 5-bromo-2'-deoxyuridine (BrdU, Sigma-Aldrich, St. Louis, MO, USA) (50 mg/kg, $10 \mathrm{mg} /$ $\mathrm{mL}$ dissolved in normal saline) twice a day for 7 consecutive days from week 3 to week 4, and then sacrificed (Fig. 3A). To assess OPC differentiation, mice were fed a CPZ-containing diet for 1 week after BrdU administration for 7 consecutive days (Fig. 4A).

\section{Morris Water Maze}

The Morris water maze test (MWM) was used to evaluate spatial location learning and memory as described in previous studies [20, 21]. All data were recorded using a computerized video system (EthoVision 3.1, Noldus Instruments, Wageningen, Gelderland, the Netherlands).

\section{Histology and Immunofluorescence}

Luxol fast blue staining and degrees of demyelination in three non-serial sections from each mouse were assessed semi-quantitatively in a blinded manner, as described previously [22]. The demyelination was scored as follows: 0 , none; 1 , rare foci; 2 , a few areas; and 3, large (confluent) areas of demyelination.

For immunofluorescence, the brain sections were blocked with $5 \%$ bovine serum albumin (BSA) and $0.3 \%$ Triton X-100 (KeyGen Biotech, Nanjing, Jiangsu, China) in $0.01 \mathrm{~mol} / \mathrm{L}$ phosphate-buffered saline (PBS) for $30 \mathrm{~min}$ at $37^{\circ} \mathrm{C}$, followed by incubation with primary antibodies overnight at $4^{\circ} \mathrm{C}$. The primary antibodies used were antiCNPase (1:200, Santa Cruz, Dallas, Texas, USA), antiMAG (1:300, Santa Cruz), anti-Nestin (1:500, Abcam, Cambridge, UK), anti-MBP (1:800, Abcam), and antiBrdU (1:500, Abcam), anti-O4 (1:200, Sigma-Aldrich, St. Louis, MO, USA), anti-Foxg1 (1:500, Sigma-Aldrich), and anti-NG2 (1:200, Millipore, Bedford, MA, USA). The sections were treated with the appropriate FITC- or TRITC-conjugated secondary antibodies (1:200, Abcam) overnight at $4^{\circ} \mathrm{C}$ and then counterstained with $4^{\prime}, 6$ diamidino-2-phenylindole (DAPI). Finally, the sections were mounted with anti-fade mounting medium. The slides for BrdU staining were pretreated with solution containing $50 \%$ formamide, $280 \mathrm{mM} \mathrm{NaCl}$, and $30 \mathrm{mM}$ sodium citrate at $65^{\circ} \mathrm{C}$ for $2 \mathrm{~h}$, incubated with $2 \mathrm{M} \mathrm{HCl}$ at $37^{\circ} \mathrm{C}$ for 30 $\mathrm{min}$, and rinsed with $0.1 \mathrm{~mol} / \mathrm{L}$ boric acid $(\mathrm{pH} 8.5)$ at room temperature for $10 \mathrm{~min}$ [2].

\section{Image Acquisition and Quantification}

Fluorescent images were captured using a confocal laser scanning microscope (Olympus FV10i, Tokyo, Japan) with appropriate excitation wavelengths. The digital images were measured using Image-Pro Plus 6.0 software (Media Cybernetics, Rockville, MD, USA). In the statistical analysis, at least 6 representative fields from randomlyselected images were acquired from each sample by a blinded observer, and then the average cell count/integrated optical density values were calculated [23]. To measure the proportion of OPCs in different differentiation stages in vitro, each dish was scanned with a $20 \times$ objective lens in $600 \mu \mathrm{m} \times 450 \mu \mathrm{m}$ format in the $x-y$ direction. The number of NG2/GFP, O4/GFP or CNPase/ GFP double-positive cells among GFP-positive cells in each field was counted by a blinded observer [2].

\section{Primary Culture of Rat OPCs}

The rat OPC proliferation culture was maintained as previously described [24, 25]. Isolated OPCs were plated at 10,000 cells $/ \mathrm{cm}^{2}$ on poly-L-lysine-coated flasks and cultured in DMEM/F12 medium supplemented with 2\% B27, $10 \mathrm{ng} / \mathrm{mL}$ platelet-derived growth factor AA (PDGF-AA, Gibco, Grand Island, NY, USA), and $10 \mathrm{~g} / \mathrm{mL}$ basic fibroblast growth factor (bFGF, Gibco) for 3 days; then, the medium was replaced with DMEM/F12 medium without PDGF-AA and bFGF for 1 day to generate preOLs. For oligodendrocyte differentiation, $10 \%$ fetal bovine serum (FBS) was added to the preOL medium and cultured for 7 days. The medium was changed every 2 days.

\section{Quantitative Real-Time Polymerase Chain Reaction (qRT-PCR)}

Genomic RNA was extracted with TRIzol reagent (Invitrogen, Carlsbad, CA, USA) according to the manufacturer's instructions and as previously described [26]. The sense and antisense primers for GSK-3 $\beta$ were as follows: forward: 5'-TCCCTCAAATTAAGGCACATC-3' and reverse: 5'-CACGGTCTCCAGTATTAGCATC-3'. The expression level of $18 \mathrm{~S}$ ribosomal RNA (rRNA) served as an internal control for the samples, and was assessed using the following sense and antisense primers: forward: 5'-CCTGGATACCGCAGCTAGG A-3' and reverse: 5'- 
- Tamoxifen administration

A Foxg1/Nestin/NG2 immunofluorescence - Morris water maze test

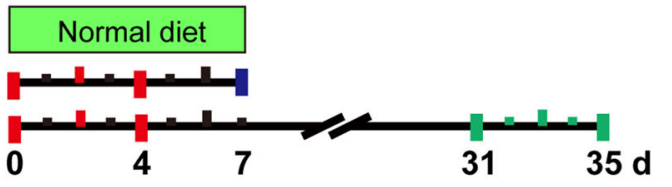

B
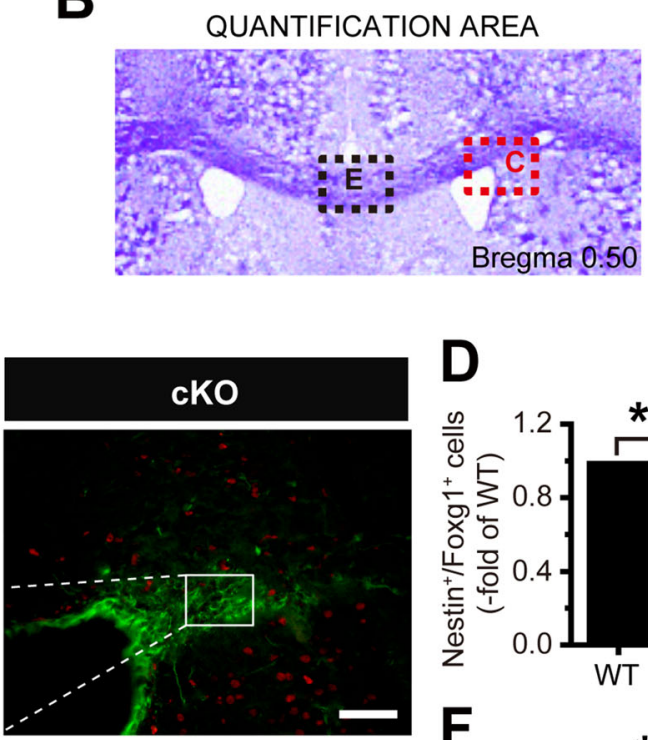

D

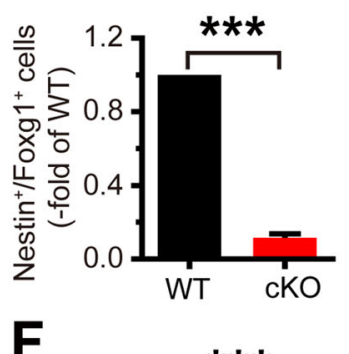

$\mathbf{F}$

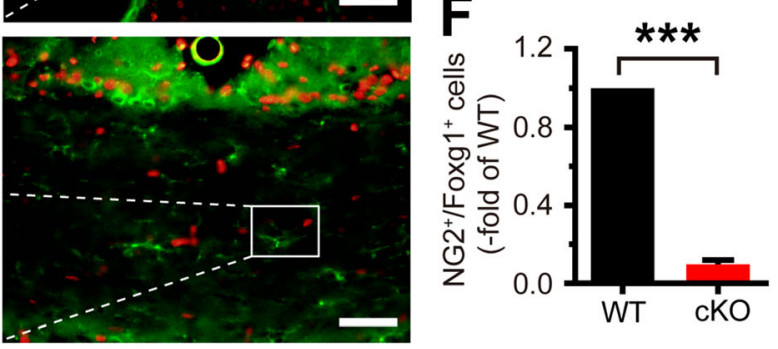

G
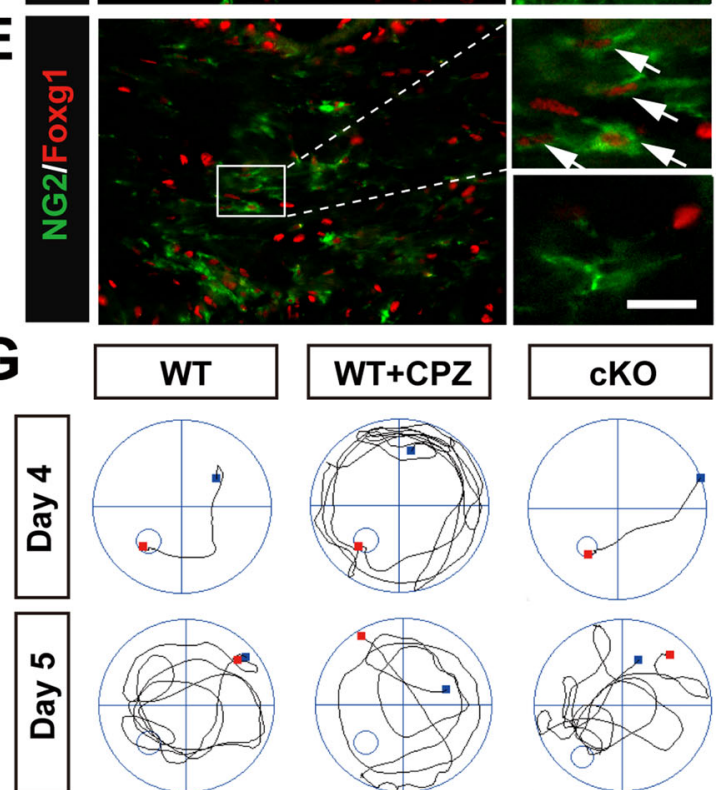

$\mathbf{H}$

(5)

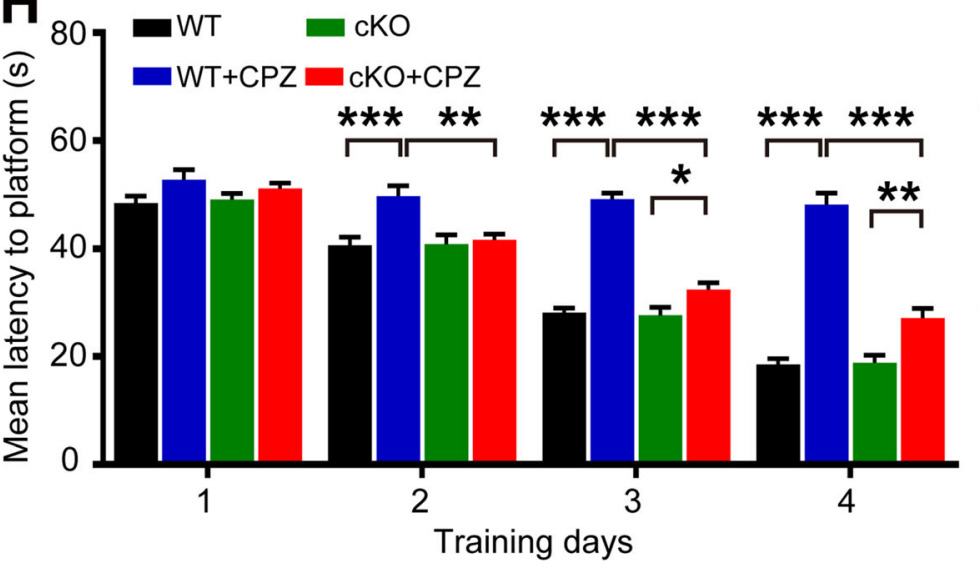

CKO+CPZ
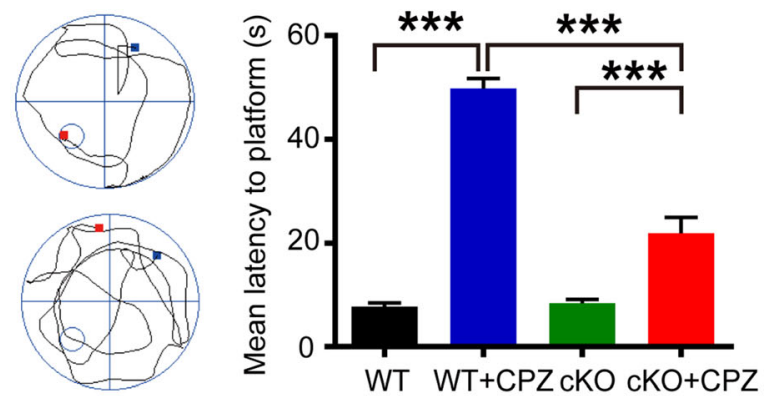
4Fig. 1 Foxg1 cKO relieves CPZ-induced learning and memory impairments in mice. A Schematic of the cuprizone (CPZ) and tamoxifen (TM) administration time points during experiment. Adult 7-week-old mice were fed a diet containing $0.2 \% \mathrm{CPZ}$ for 5 weeks (demyelination phase); TM was intraperitoneally injected ( 3 times, $24 \mathrm{~h}$ apart) from day 0 after feeding the CPZ diet; mice were sacrificed immediately after the Morris water maze (MWM) test. B Image showing the measured region in the coronal sections in $\mathbf{C}, \mathbf{D}$ (red dashed box) and $\mathbf{E}, \mathbf{F}$ (black dashed box). $\mathbf{C}$, D Effect of Foxg1 knockout on neural stem cells assessed by Nestin/ Foxg1 immunofluorescence staining $(\mathbf{C})$ and quantitative analysis $(\mathbf{D})$. E, F Effect of Foxgl knockout on oligodendrocyte precursor cells assessed by NG2/Foxg1 immunofluorescence staining (E) and quantitative analysis $(\mathbf{F})$. G Representative tracking from each group of mice in the MWM test on days 4 and 5 (small circles, location of the platform; blue and red points, start and end locations of the mouse, respectively). H Average latency to find a hidden platform during the first 4 days of training in the directional navigation experiment. I Latency to find the platform on day 5 when the platform was removed. J Number of crossings in the spatial exploration experiment on day 5 of the MWM (white arrows in $\mathbf{C}$ and $\mathbf{E}$, representative double-labeled positive cells). $n=8$ per group. Data are presented as the mean \pm SEM. $* P<0.05$, $* * P<0.01$, $* * * P<$ 0.001 , Student's $t$-test in $\mathbf{D}, \mathbf{F}$; one-way ANOVA with Tukey's post hoc test in $\mathbf{H}-\mathbf{J}$. Scale bars, $25 \mu \mathrm{m}$ in $\mathbf{C}, \mathbf{E} ; 10 \mu \mathrm{m}$ in the enlarged images.

GCGGCGCAATACGAATGCCCC- $3^{\prime}$. The relative level of Foxg 1 mRNA expression was calculated according to the standard $2^{-\Delta \Delta \mathrm{Ct}}$ method by normalization to the $18 \mathrm{~S}$ rRNA mRNA level.

\section{Western Blot Analysis}

Western blot analysis was performed as previously described [27, 28]. Total protein was extracted from cultured rat OPCs using cell lysis buffer supplemented with proteinase and phosphatase inhibitors. The nuclear proteins were extracted using a commercial kit (Thermo Fisher Scientific, Waltham, MA, USA). Protein samples were separated on $10 \%$ SDS-PAGE gels and transferred to nitrocellulose membranes. The primary antibodies were anti-Foxg1 (1:800, Sigma-Aldrich), anti-GSK-3 $\beta$ (1:600, Sigma-Aldrich), anti- $\beta$-actin (1:1000, Santa Cruz), anti- $\beta$ catenin (1:500, Abcam), and anti-histone H3 (1:1000, Sigma-Aldrich). The band intensity was quantified using ImageJ software (NIH, Bethesda, MD, USA). Values were normalized to the $\beta$-actin/histone $\mathrm{H} 3$ level.

\section{Lentiviral Vector Production}

A lentivirus encoding small hairpin RNA (shRNA) for Foxg1 was designed and synthesized by GeneChem Co., Ltd (Shanghai, China). The Foxg1 locus on chromosome 2 and its 200-bp flanking sequences were amplified by PCR from genomic rat DNA and inserted into the GV248 vector (the functional element is Ubi-EGFP-MCS-IRES-puromy- cin). The shRNA sequence for Foxgl was $5^{\prime}$ TCGGGCCAAGCTAGCCTTTAA- $3^{\prime}$. To generate the construct, the scrambled sequence $5^{\prime}$ TTCTCCGAACGTGTCACGT-3' was inserted. In the scrambled sequence, the nucleotides were randomly added with no target sequence tracks. Preparations of the recombinant lentivirus were made by transient co-transfection of HEK 293T cells accompanied by proper transfer vectors and lentiviral helper plasmids $(20 \mu \mathrm{g}$ of pGC-LV, $15 \mu \mathrm{g}$ of pHelper 1.0, and $10 \mu \mathrm{g}$ of pHelper 2.0) using Lipofectamine 2000 (Invitrogen, Waltham, MA, USA) according to the manufacturer's instructions. OPCs were transfected with lentiviral Foxgl shRNA $72 \mathrm{~h}$ before experiments.

\section{EdU Labeling of OPCs}

For EdU labeling, $3.5 \times 10^{4}$ cells were seeded into each well of a 48-well plate in DMEM/F-12 medium (1:1) supplemented with 10\% FBS. Twenty-four hours later, EdU (RiboBio, Guangzhou, China) was added to the medium at $10 \mu \mathrm{mol} / \mathrm{L}$. Another $24 \mathrm{~h}$ later, the cells were fixed with $4 \%$ paraformaldehyde for $30 \mathrm{~min}$. For EdU staining, the cells were then incubated with freshly-made $1 \times$ Apollo $^{\circledR} 567$ reaction cocktail for $30 \mathrm{~min}$ at room temperature in the dark. Then, the cells were incubated in $0.5 \%$ Triton X-100 in PBS for $10 \mathrm{~min}$ at room temperature. After washing twice with PBS, the cells were fixed in methanol and then stained with Hoechst 33342. The percentage of EdU-positive cells was calculated as the number of Apollo $^{\circledR} 567$-stained cells/the number of Hoechst 33342-stained cells.

\section{Statistical Analysis}

Data were analyzed with SPSS software version 19.0 (IBM, Armonk, NY, USA) and are expressed as the mean \pm SEM. $T$-tests or one-way analysis of variance (ANOVA) followed by the Newman-Keuls or Tukey's HSD post tests were used for comparisons between two or multiple groups, respectively. Statistical significance was set at $P$ $<0.05$.

\section{Results}

\section{Conditional Ablation of Foxg1 Ameliorates Behav- ioral Deficits and Demyelination Induced by CPZ}

On the first day (day 0) of CPZ treatment, the mice were intraperitoneally injected with TM to induce Foxgl gene 
knockout (Fig. 1A). The results showed that, compared with control littermates, Foxg1 expression on neural stem cells in the subventricular zone (Fig. 1C, D) or OPCs in the corpus callosum region (Fig. 1E, F) was significantly reduced in Foxgl-cKO mice on day 7 after TM injection. Studies have shown that $\mathrm{CPZ}$ administration results in a decline in learning and memory in mice [29, 30]. The MWM test showed that Foxgl cKO significantly shortened the prolonged mean latency from day 2 to day 4 (Fig. $1 \mathrm{G}$, $\mathrm{H}$ ), and it significantly decreased the latency to the platform on day 5 (Fig. 1G, I). The crossing number of Foxg1-cKO mice was clearly increased compared with the $\mathrm{WT}+\mathrm{CPZ}$ mice (Fig. 1J). These results suggested that Foxgl cKO significantly alleviates the learning and memory impairments induced by $\mathrm{CPZ}$ in mice.

LFB staining showed that myelin was intact in the corpus callosum of WT mice, but it had almost entirely disappeared in the WT+CPZ mice (Fig. 2A). The demyelination score was significantly lower in the Foxgl
$\mathrm{cKO}+\mathrm{CPZ}$ group than in the $\mathrm{WT}+\mathrm{CPZ}$ group (Fig. 2D). The statistical data from the myelin basic protein (MBP) immunofluorescence staining was consistent with the LFB staining results (Fig. 2B, E). These results indicated that demyelination induced by $\mathrm{CPZ}$ is significantly relieved by the absence of Foxgl.

\section{Foxg1 cKO Decreases Proliferation but Promotes the Differentiation of OPCs in CPZ-Induced Demyelination}

BrdU labeling was used to assess cell proliferation (Fig. 3A) and differentiation (Fig. 4A) in the corpus callosum, and the results showed that the number of BrdU-positive cells was significantly lower in the Foxgl $\mathrm{cKO}+\mathrm{CPZ}$ group than the $\mathrm{WT}+\mathrm{CPZ}$ group (Fig. $3 \mathrm{C}, \mathrm{D}$ ). Furthermore, the increase in percentage of $\mathrm{NG}^{+}$cells (Fig. 3E, F) and $\mathrm{O}^{+}$cells (Fig. 3J) cells in the $\mathrm{WT}+\mathrm{CPZ}$ group was prevented by Foxgl-cKO treatment. The
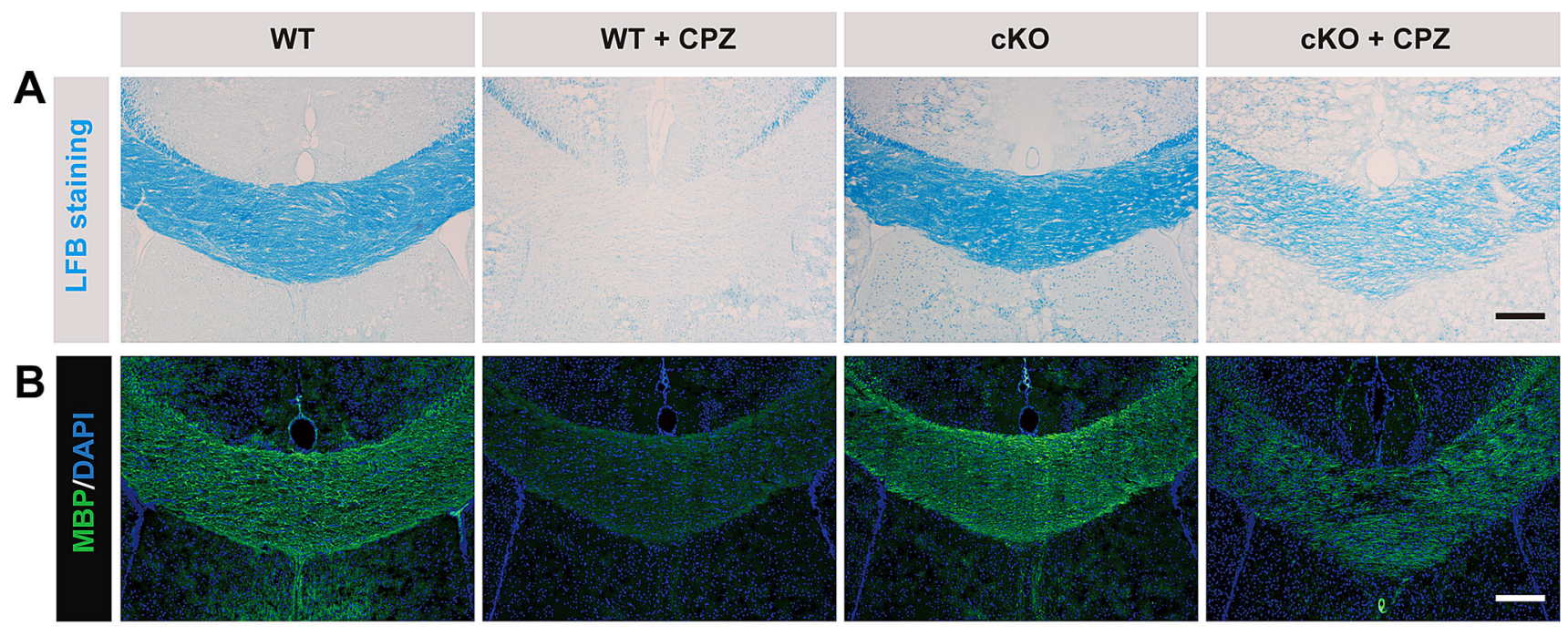

C

D
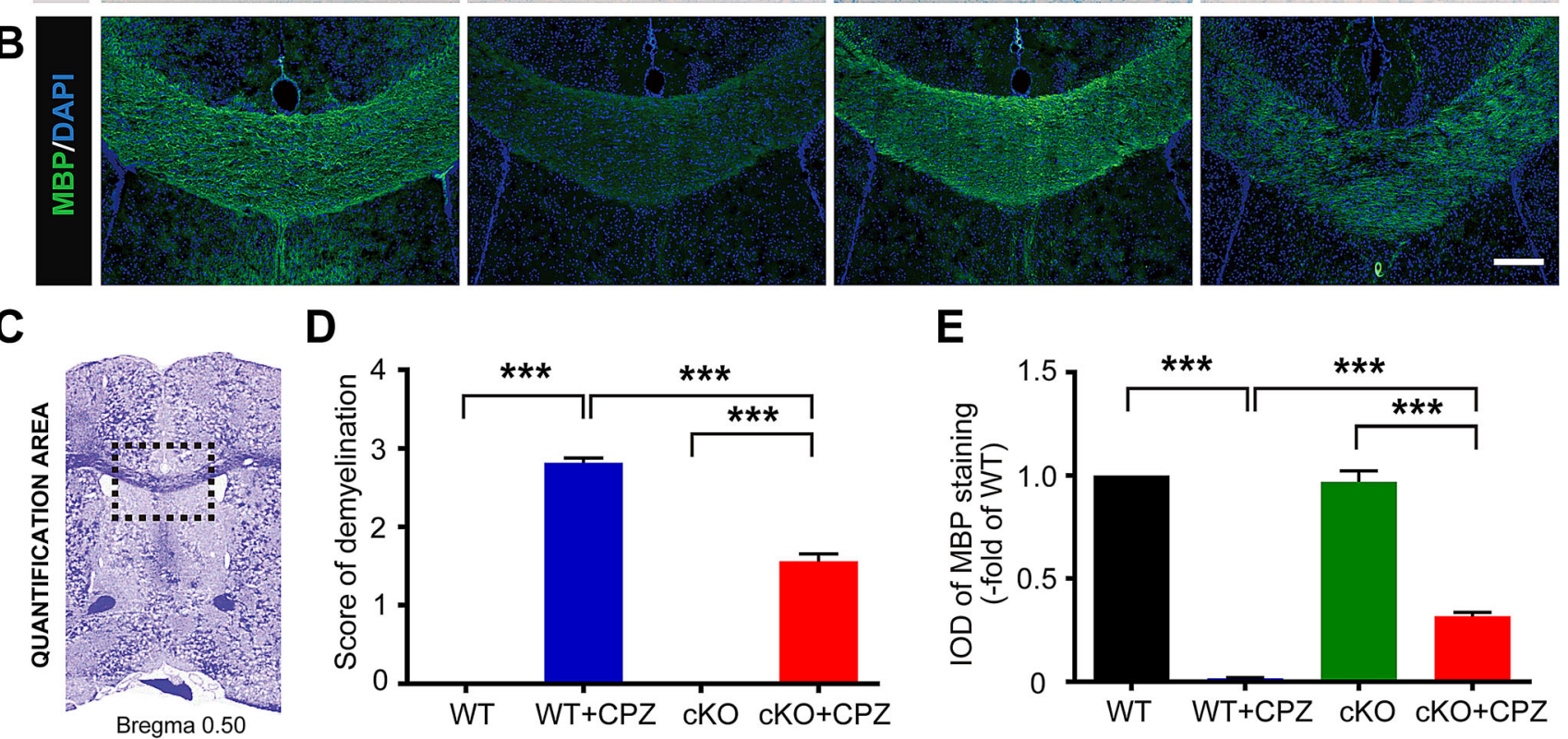

E

Fig. 2 Conditional ablation of Foxg1 alleviates demyelination induced by cuprizone (CPZ) in mice. A Representative images of LFB staining showing demyelination in the corpus callosum (CC) of each group. B Representative images of NG2 immunofluorescence staining in the $\mathrm{CC}$ of each group. $\mathbf{C}$ Image showing the measured region (black dashed box) in the coronal sections in $\mathbf{A}, \mathbf{B}$. and $\mathbf{D}$ The degree of demyelination was analyzed in sections as in A. E Quantification of the MBP fluorescence measured as integrated optical density (IOD) in each group from sections as in $\mathbf{B} . n=8$ per group; data are presented as the mean $\pm \mathrm{SEM}$; $* * * P<0.001$, one-way ANOVA with Tukey's post hoc test; scale bars, $100 \mu \mathrm{m}$. 
A

- Tamoxifen administration

- BrdU injection
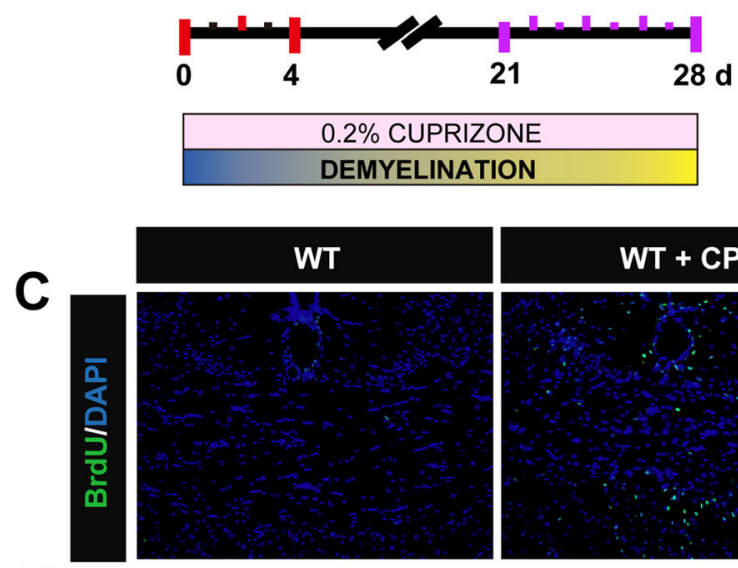

E

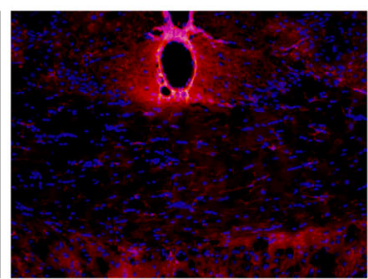

G
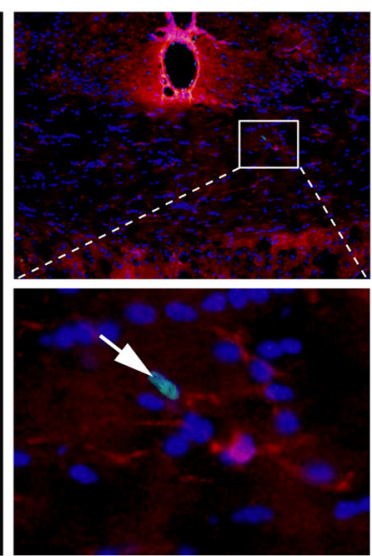

I

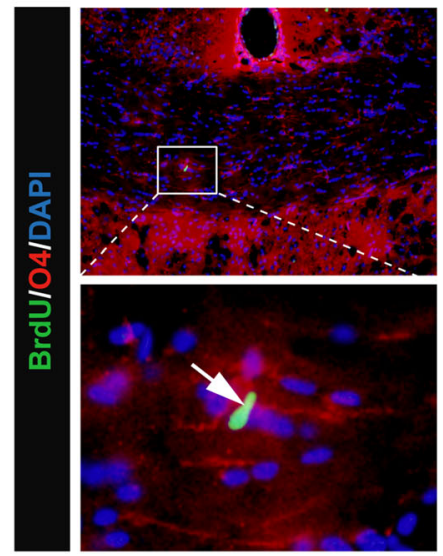

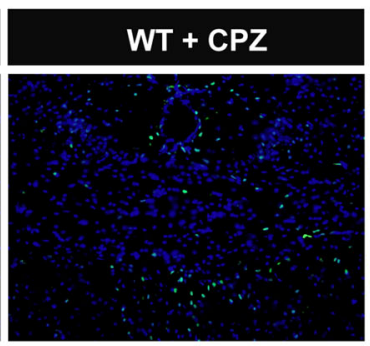
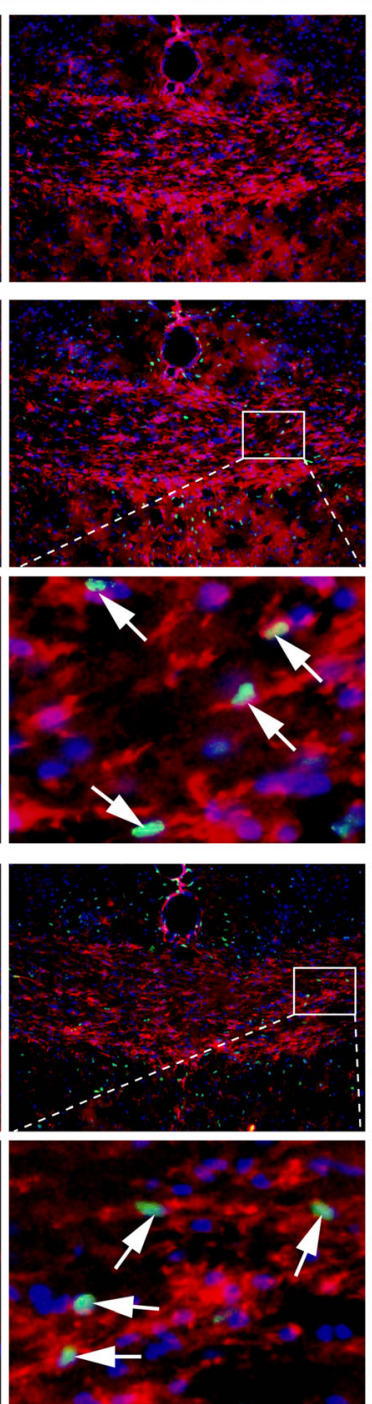

B
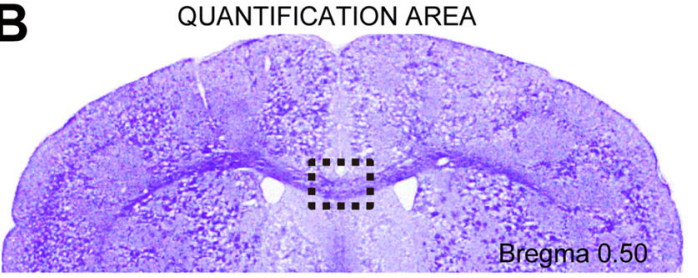

$\mathrm{cKO}+\mathrm{CPZ}$
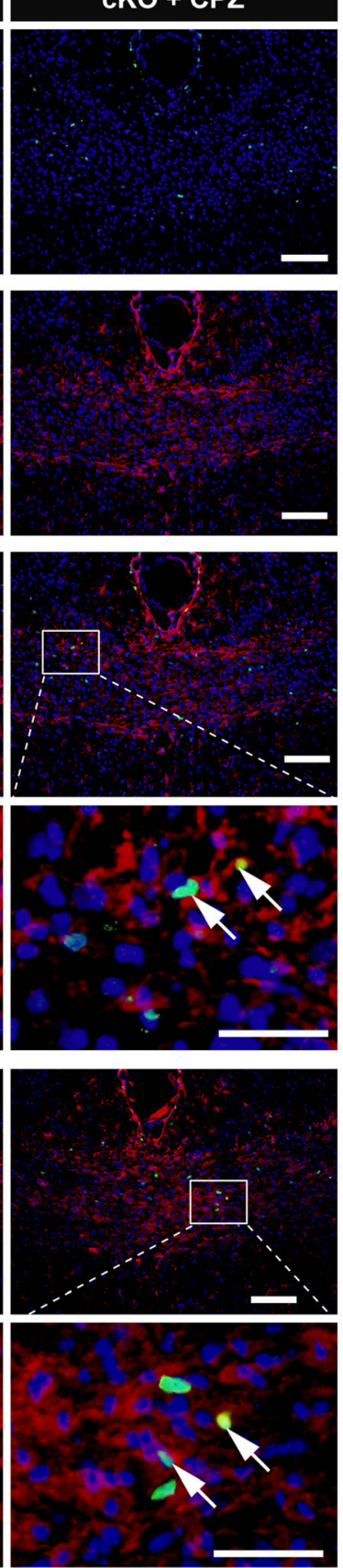
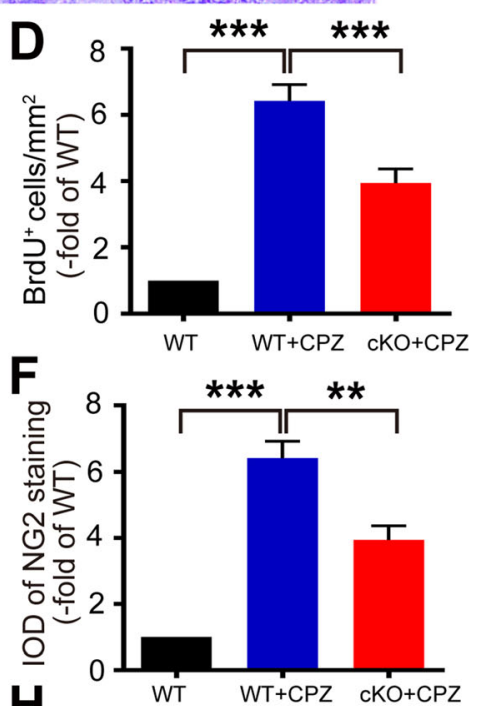

H
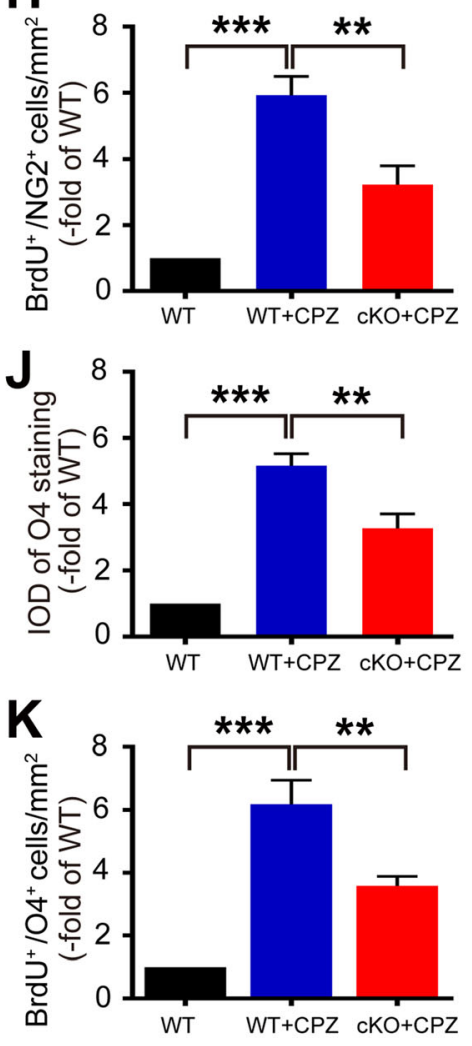
4Fig. 3 Conditional knockout of Foxg1 decreases the proliferation of oligodendrocyte precursor cells in the corpus callosum (CC) of mice induced with cuprizone (CPZ). A Schematic of CPZ and BrdU administration time points during the experiment. Adult 7-week-old mice were fed a diet containing $0.2 \%$ CPZ for 4 weeks (demyelination phase) to assess the proliferation of intrinsic OPCs; Tamoxifen (TM) was intraperitoneally injected ( 3 times, $24 \mathrm{~h}$ apart) from day 0 after starting the CPZ diet; BrdU was intraperitoneally injected twice a day for 7 consecutive days, and then the mice were sacrificed. B Image showing the measured region (black dashed box) in coronal sections as in $\mathbf{C}-\mathbf{K}$. C Immunofluorescence staining of BrdU in the CC of each group. D Quantitative analysis of $\mathrm{BrdU}^{+}$cells as in C. E Immunofluorescence staining of $\mathrm{NG} 2$ in the $\mathrm{CC}$ of each group. F Quantitative analysis of $\mathrm{NG}^{+}$cells as in E. G, $\mathbf{H}$ Immunofluorescence staining of BrdU/NG2 $(\mathbf{G})$ and quantitative analysis $(\mathbf{H})$ in the $\mathrm{CC}$ of each group. I Immunofluorescence staining of BrdU/O4 in the $\mathrm{CC}$ of each group. $\mathbf{J}, \mathbf{K}$ Quantitative analysis of $\mathrm{O}^{+}$cells $(\mathbf{J})$ and $\mathrm{BrdU}^{+} / \mathrm{O}^{+}$cells as in $\mathbf{I}$. White arrows, representative double-labeled positive cells; $n=8$ per group; data are presented as the mean \pm SEM; $* * P<0.01, * * * P<0.001$, one-way ANOVA with Tukey's post hoc test; scale bars, $50 \mu \mathrm{m}$ in $\mathbf{C}, \mathbf{E}$ and the upper panels in $\mathbf{G}, \mathbf{I}$; $20 \mu \mathrm{m}$ in the lower panels of $\mathbf{G}, \mathbf{I}$.

number of proliferating OPCs, indicated by $\mathrm{NG}^{+} / \mathrm{BrdU}^{+}$ (Fig. 3G, H) or $\mathrm{BrdU}^{+} / \mathrm{O}^{+}$(Fig. 3I, K), was also decreased; however, the number of new mature oligodendrocytes labeled with $\mathrm{BrdU}^{+} / \mathrm{MBP}^{+}$(Fig. 4C, D), $\mathrm{BrdU}^{+} /$ $\mathrm{CNPase}^{+}$(Fig. 4E, F), and $\mathrm{BrdU}^{+} / \mathrm{MAG}^{+}$(Fig. 4G, H) differentiated from the OPCs was significantly higher in the Foxgl cKO+CPZ group than in the WT+CPZ group. These data suggested that Foxgl cKO decreases the production or proliferation of OPCs but promotes their differentiation.

\section{Foxg1 cKO Facilitates Myelination and the Recov- ery of Cognitive Function During Remyelination}

To study the role of Foxgl in the regeneration of OLs after CPZ-induced myelin sheath injury, TM was administered on day 1 of CPZ withdrawal (Fig. 5A). Behavioral results showed that the average latency was significantly longer in the WT+CPZ than the WT group; however, Foxg1 deletion in CPZ-treated mice clearly decreased the latency (Fig. 5B, C). In the spatial exploration experiment on day 5 , the number of platform crossings was significantly higher in the Foxg $1 \mathrm{cKO}+\mathrm{CPZ}$ than the WT+CPZ group (Fig. 5D). These results suggested that Foxgl cKO significantly promotes the recovery of learning and memory after $\mathrm{CPZ}$ withdrawal in mice.

After 2 weeks of feeding without CPZ, the demyelination score was decreased (Fig. 5F, G) and the fluorescence intensity of MBP significantly enhanced (Fig. 5H, I) in the Foxgl-cKO mice. These results indicated that Foxgl deficiency favors myelin repair after CPZ-induced demyelinating injury.

\section{Foxg1 cKO Inhibits Proliferation but Promotes the Differentiation of OPCs In Vitro}

In vitro, GFP-tagged shRNA knockdown of Foxgl using lentiviral transfection was required to confirm its function in the proliferation and differentiation of cultured OPCs. The results of immunofluorescence staining (Fig. 6A, B) and Western blotting (Fig. 6D) demonstrated that Foxg 1 knockdown strongly decreased the expression of Foxg1 in primary cultured rat OPCs. The lentiviral transfection efficiency was defined by GFP fluorescence (Fig. 6C). The results of EdU staining showed that the number of $\mathrm{EdU}^{+}$ cells was significantly smaller in the Foxgl shRNA than the control group (Fig. 6E, F). These results indicated that, to a certain extent, knockdown of Foxgl decreases the proliferation of OPCs in vitro, suggesting that Foxgl participates in the regulation of OPC proliferation.

To confirm whether Foxg1 regulates OPC differentiation, we downregulated the level of Foxgl in OPCs and analyzed the number of immature and mature OLs. In contrast to the control group, most of the cells in the shRNA group were labeled with CNPase (Fig. 6I), and only a few were labeled with NG2 (Fig. 6G) or O4 (Fig. 6H). The number of O4-positive cells was significantly lower and CNPase-positive cells significantly higher in the Foxgl shRNA than in the Ctrl group (Fig. 6J), suggesting that Foxgl plays a negative regulatory role during OPC differentiation into OLs.

\section{Foxg1 May Participate in the Proliferation and Dif- ferentiation of OPCs Through the Wnt Signaling Pathway}

To explore whether Foxgl influences Wnt signal activation, we assessed the expression of GSK-3 $\beta$ and $\beta$-catenin. The results showed that the level of GSK-3 $\beta$ mRNA (Fig. 7A) and protein (Fig. 7B) was significantly higher in the Foxgl shRNA than in the control group, while the expression of $\beta$-catenin was significantly decreased in Foxg 1 -knockdown OPCs (Fig. 7C). The GSK-3 $\beta$ inhibitor SB216763 reversed the decrease in $\beta$-catenin induced by Foxg1 knockdown (Fig. 7C). Moreover, SB216763 treatment also clearly increased the number of $\mathrm{EdU}^{+}$cells (Fig. 7D, E) and the proportions of $\mathrm{NG}^{+}$(Fig. 7F, I) and $\mathrm{O}^{+}$cells (Fig. 7G, I) and decreased the proportion of CNPase (Fig. 7H, I) when compared with the Foxgl shRNA group. These results indicated that Foxgl might be involved in the proliferation and differentiation of OPCs through the Wnt signaling pathway. 
A Iamoxifen administration

- BrdU injection
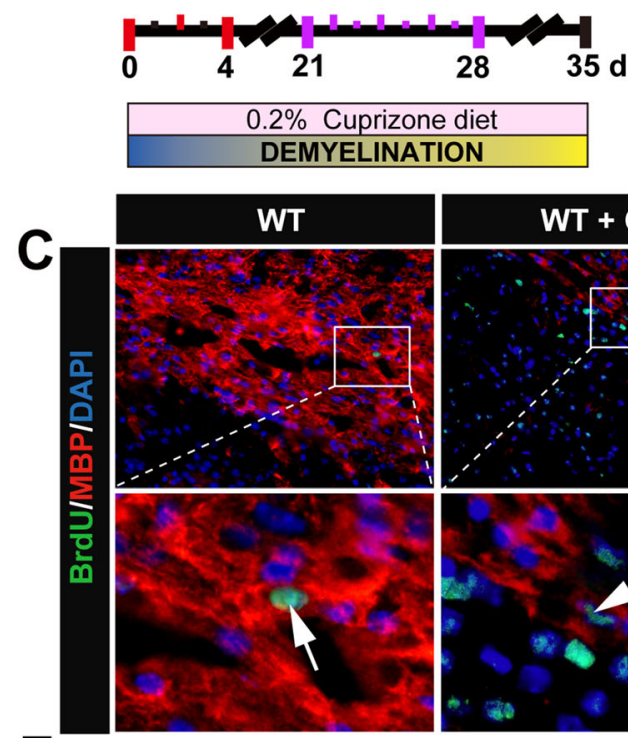

E

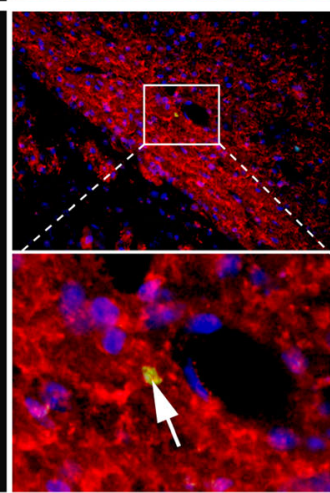

$G$

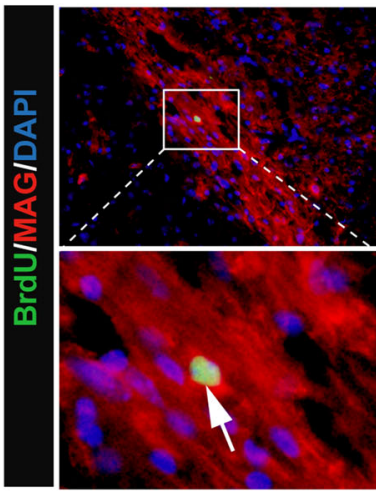

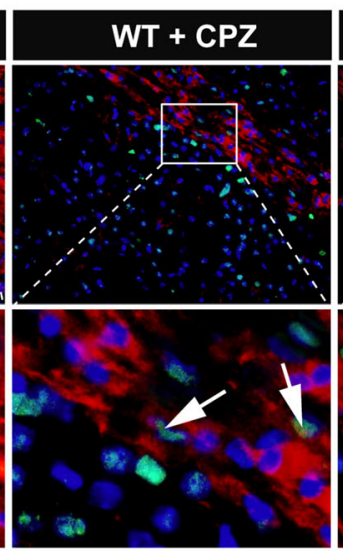
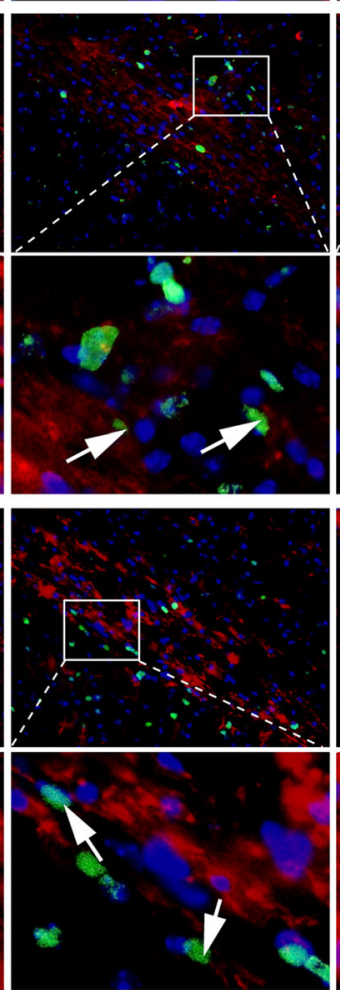

B
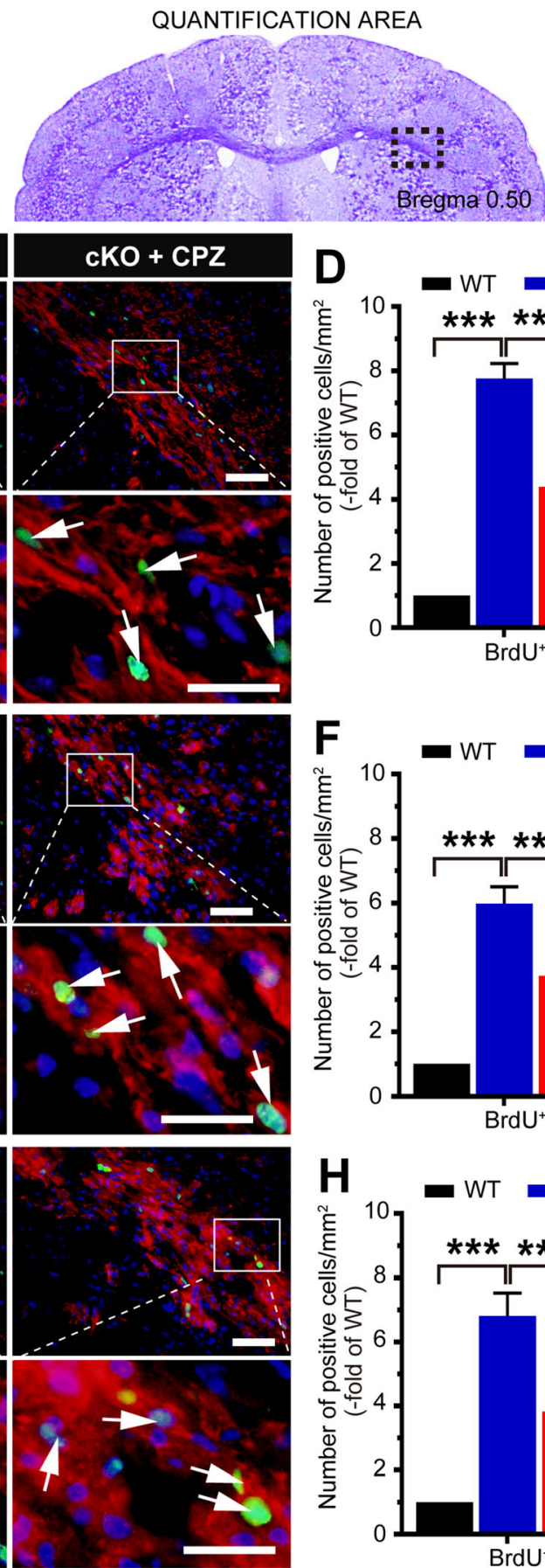
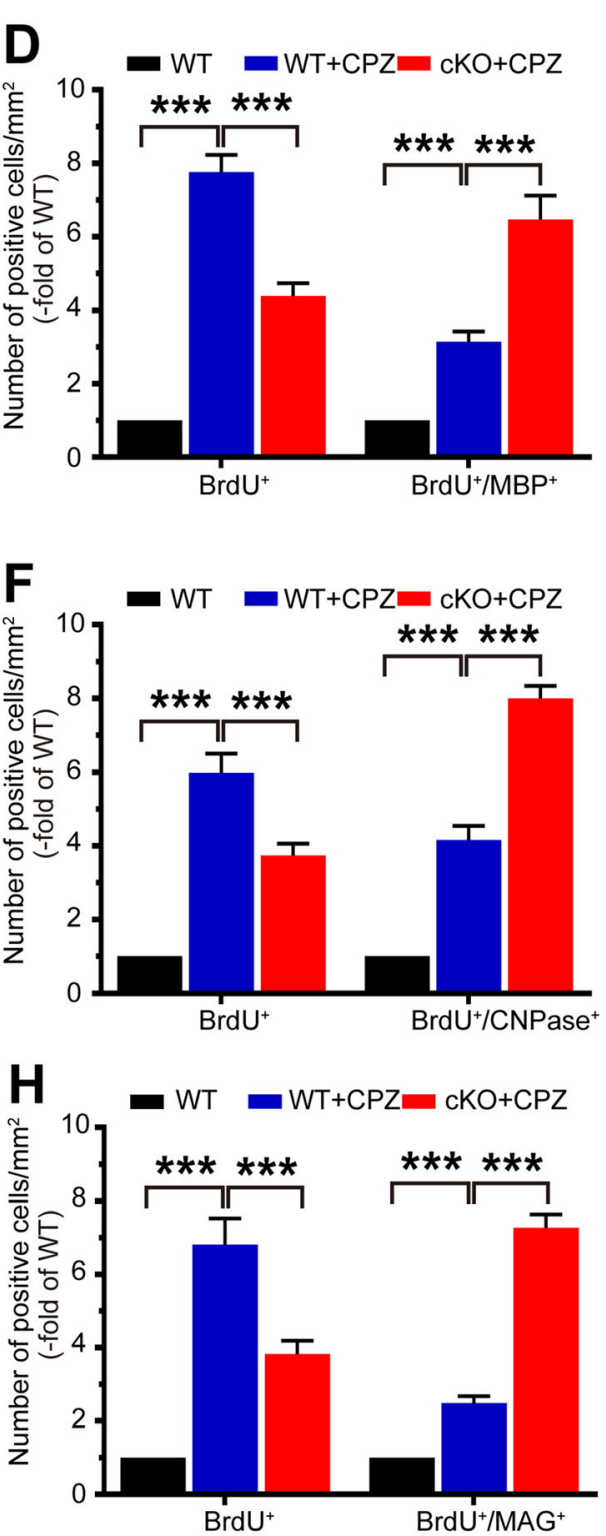

Fig. 4 Conditional knockout of Foxg1 promotes the differentiation of oligodendrocyte precursor cells in the corpus callosum (CC) of mice induced by CPZ. A Schematic of cuprizone (CPZ) and BrdU administration time points during the experiment. Adult 7-week-old mice were fed a diet containing $0.2 \% \mathrm{CPZ}$ for 5 weeks (demyelination phase); Tamoxifen (TM) was intraperitoneally injected (3 times, $24 \mathrm{~h}$ apart) from day 0 after feeding on a CPZ diet; BrdU was intraperitoneally injected twice a day for 7 consecutive days, and the mice were sacrificed on day 35 . B Image showing the measured region (black dashed box) in coronal sections as in $\mathbf{C}-\mathbf{H}$. $\mathbf{C}-$ H Immunofluorescence double-labeled staining and quantitative analysis of BrdU/MBP $(\mathbf{C}, \mathbf{D}), \operatorname{BrdU} / \mathrm{CNPase}(\mathbf{E}, \mathbf{F})$, and BrdU/ MAG $(\mathbf{G}, \mathbf{H})$ in the CC of Foxg1-cKO mice. White arrows indicate representative double-labeled positive cells; $n=8$ per group; data are presented as the mean $\pm \mathrm{SEM} ; * * P<0.01$, $* * * P<0.001$, one-way ANOVA with Tukey's post hoc test; scale bars, $20 \mu \mathrm{m}$ in the upper panel; $10 \mu \mathrm{m}$ in the lower panel. 
A

- Tamoxifen administration

- Morris water maze test

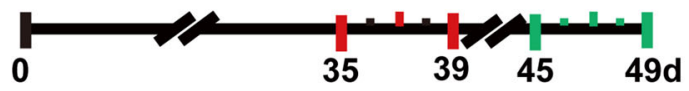

\begin{tabular}{|c|c|}
\hline $0.2 \%$ Cuprizone diet & Normal diet \\
\hline DEMYELINATION & REMYELINATION \\
\hline
\end{tabular}
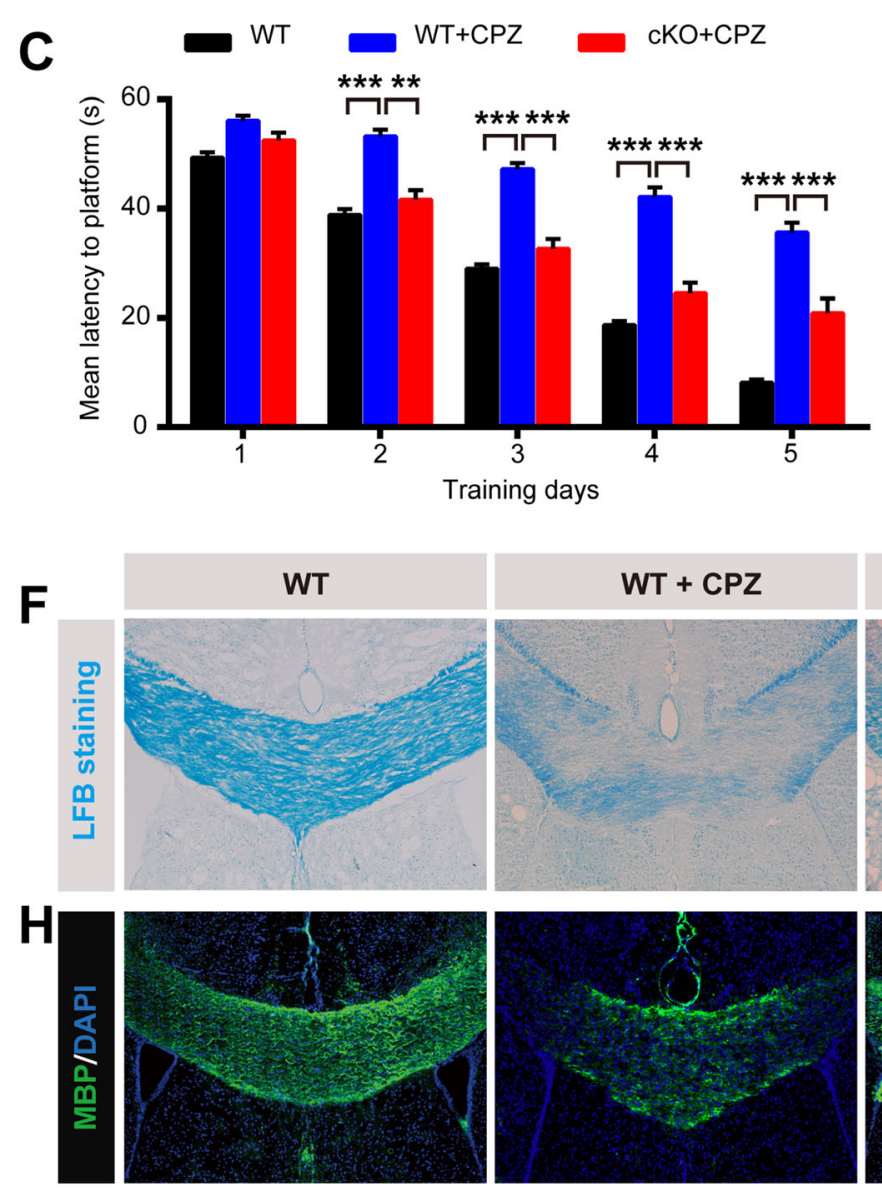

Fig. 5 Foxg 1 cKO promotes the recovery of cognitive function and remyelination after $\mathrm{CPZ}$ withdrawal in mice. A Timeline of the experimental design. Adult 7-week-old mice were fed a diet containing $0.2 \%$ cuprizone (CPZ) for 5 weeks (demyelination phase), followed by a normal diet for 2 weeks (remyelination phase); Tamoxifen (TM) was intraperitoneally injected (3 times, $24 \mathrm{~h}$ apart) after CPZ withdrawal; mice were sacrificed immediately after the Morris water maze (MWM) test. B Representative tracking in each group in the MWM test on days 4 and 5 (small circles, location of the platform; blue and red points, start and end locations, respectively). C Average latency for each group to find a hidden platform over the
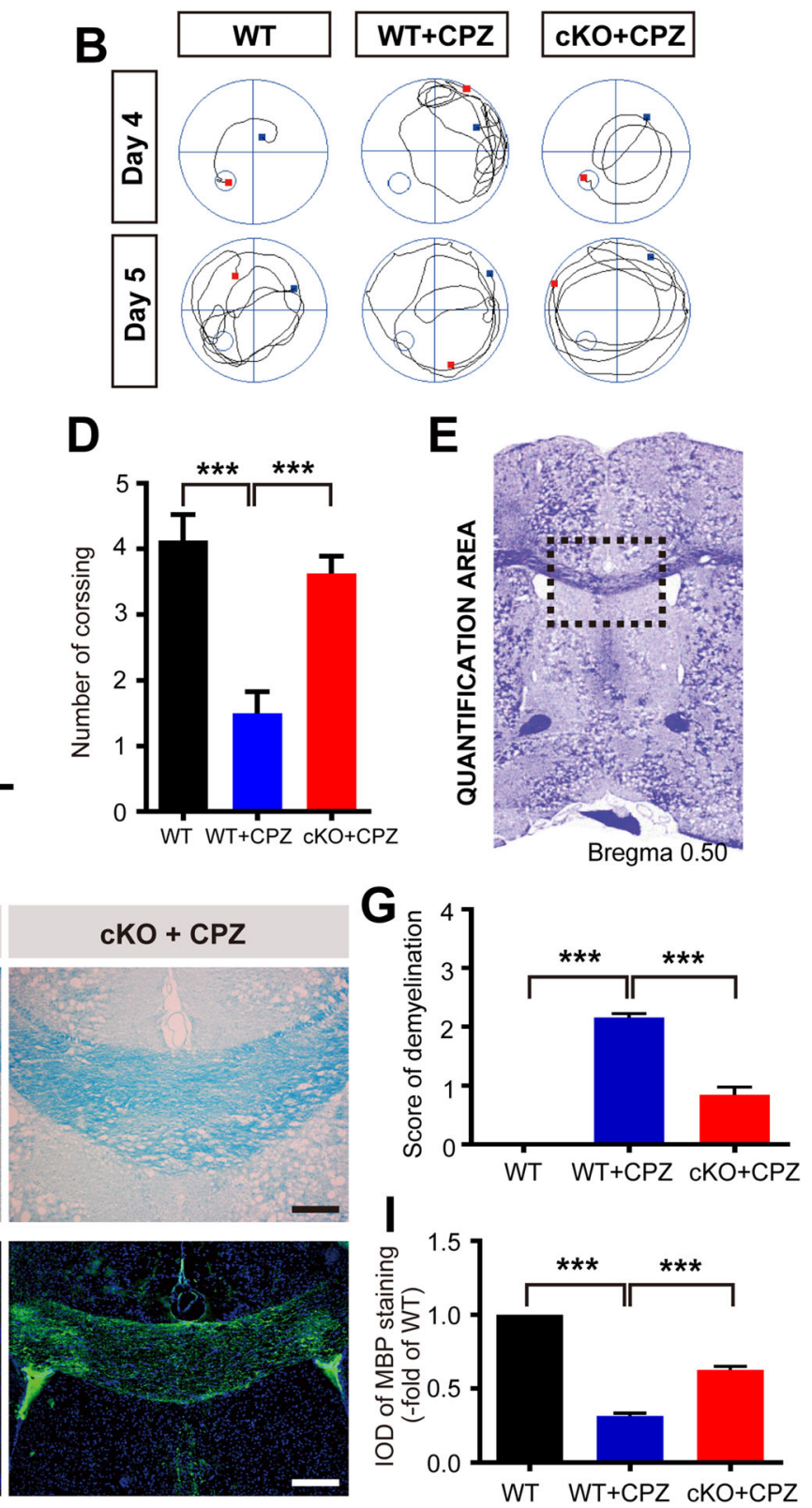

first 4 days of training in the directional navigation experiment and on day 5 when the platform was removed. D Number of crossings in each group in the spatial exploration experiment on day 5 of the MWM. E Image showing the measured region (black dashed box) in coronal sections as in $\mathbf{F}-\mathbf{I}$. F Representative images of LFB staining for demyelination in the corpus callosum in each group. G Analysis of the degree of demyelination as in F. H, I MBP immunofluorescence (H) and analysis of integrated optical density (IOD) (I) in each group. $n=8$ per group; data are presented as the mean $\pm \mathrm{SEM} ; * * P<0.01$, *** $P<0.001$, one-way ANOVA with Tukey's post hoc test; scale bars, $50 \mu \mathrm{m}$. 

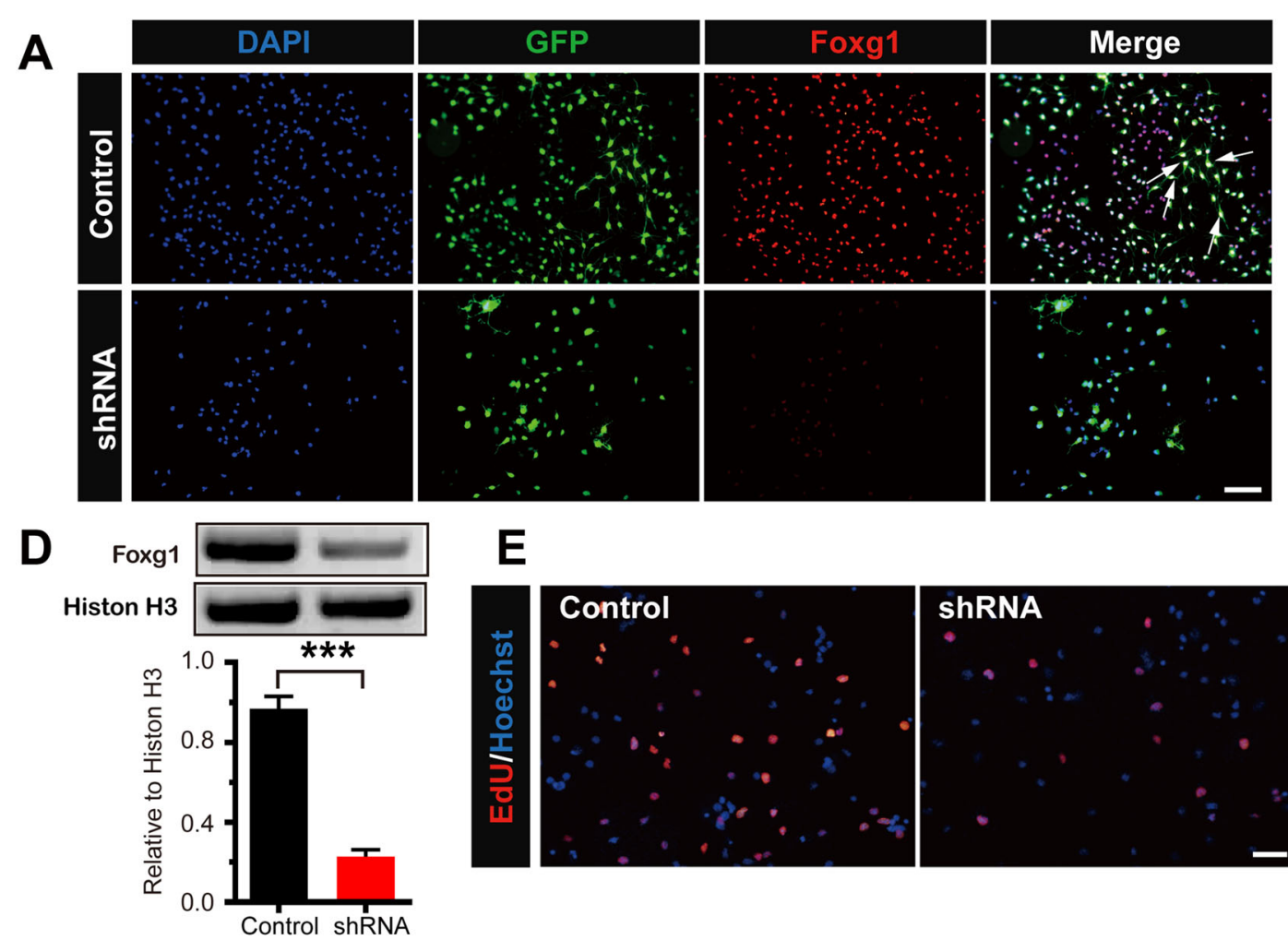

\section{E}
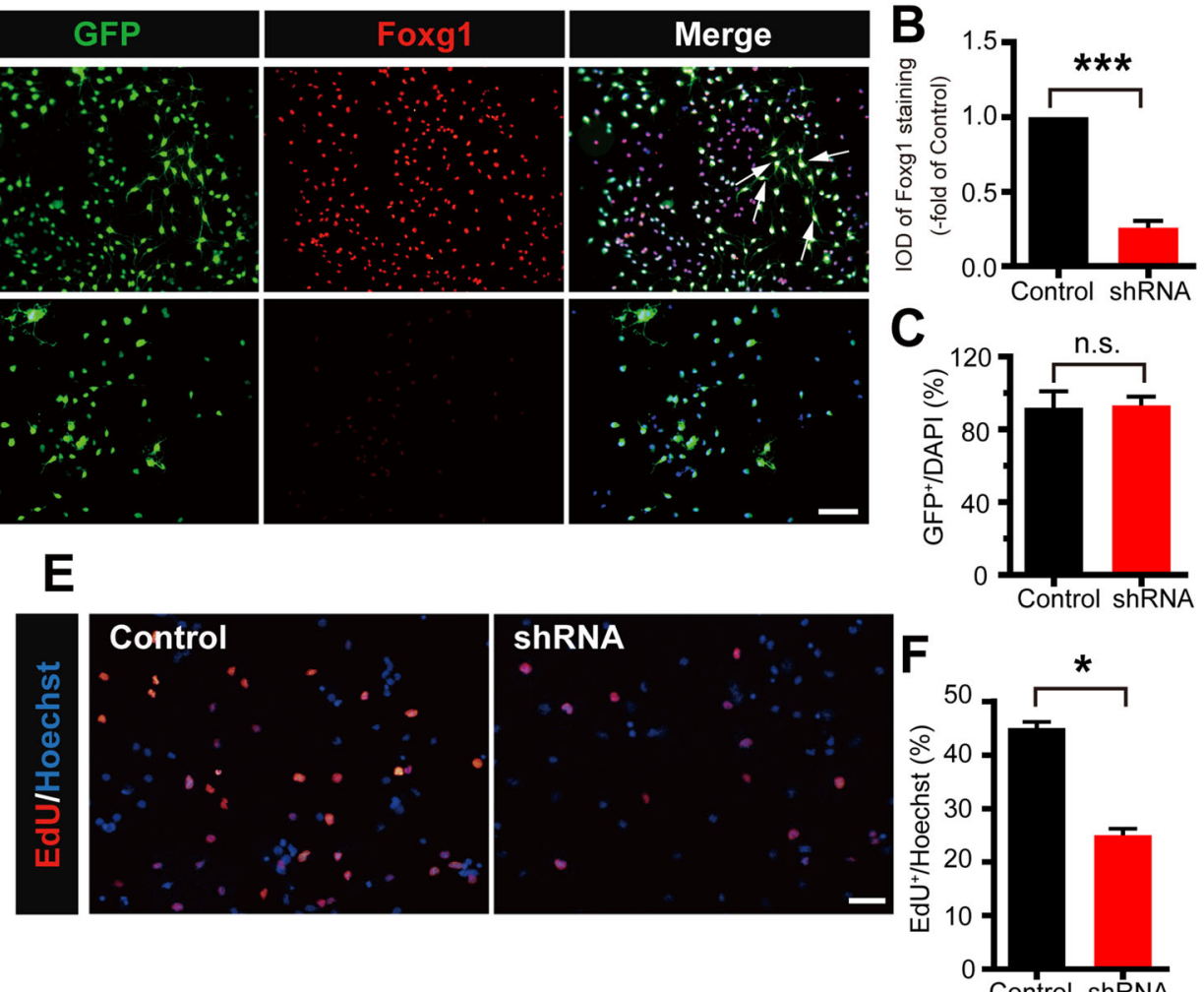

C
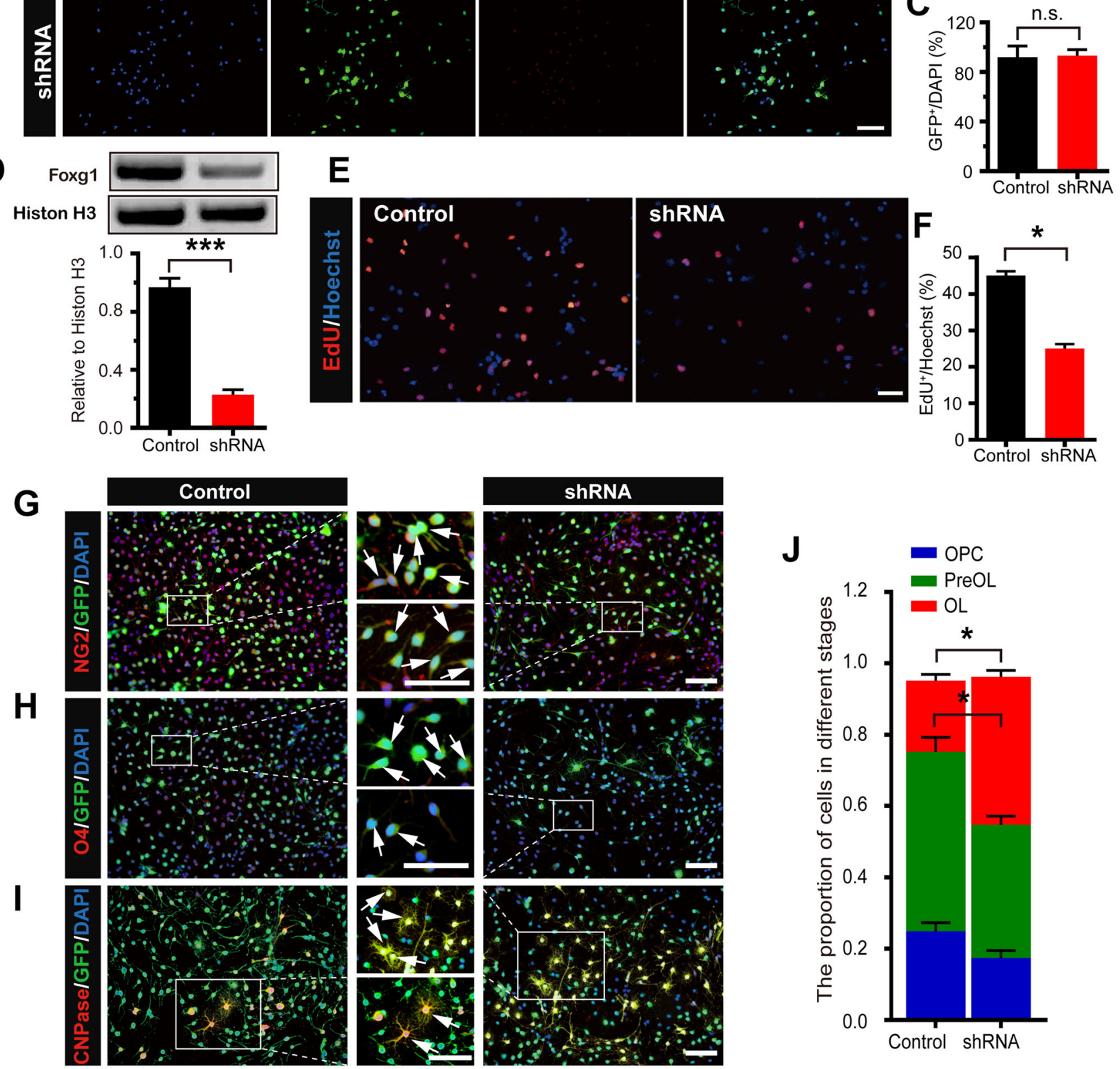

Fig. 6 Knockdown of Foxg1 decreases proliferation while promoting the differentiation of OPCs in vitro. A Foxg1 knockdown decreases the expression of Foxg1 in primary cultured rat OPCs. Cells transfected with a lentiviral GFP-tagged vector inserted into a scrambled sequence (Control) or lentiviral GFP-tagged Foxg 1 shRNA (shRNA) for 3 days and immunostained with anti-Foxg1 antibody. B Quantification of Foxg1 fluorescence integrated optical density (IOD) as in A. C The transfection efficiency as defined by GFP fluorescence. D Western blots and quantitative analyses of
Foxg1 protein expression in primary cultured rat OPCs. E, F Representative images of EdU-positive proliferating cells (E) and quantitative analysis (F) of Control and Foxg1 shRNA-treated OPCs. GI Representative images of NG2 (G), O4 (H), and CNPase (I) staining in each group of cells. $\mathbf{J}$ Cell proportions at different stages during the differentiation of OPCs. White arrows, representative doublelabeled positive cells; $n=6$ per group; data are presented as the mean \pm SEM; ${ }^{*} P<0.05, * * * P<0.001$, n.s. not significant, Student's $t$ test; scale bars, $40 \mu \mathrm{m}$. 
A
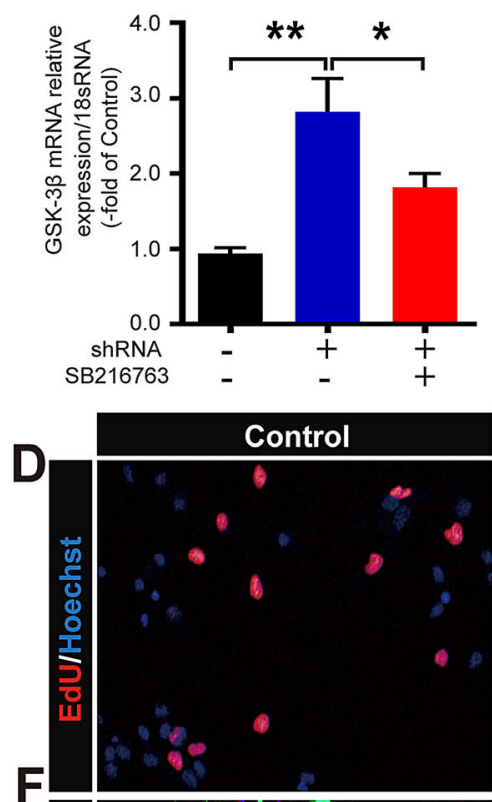

$\mathbf{F}$

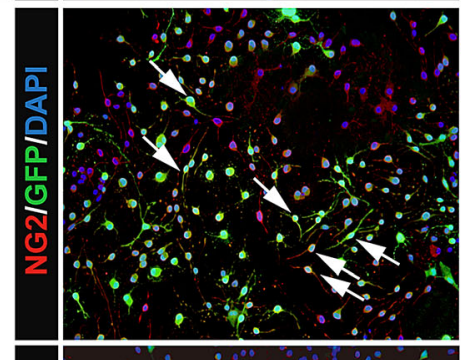

G

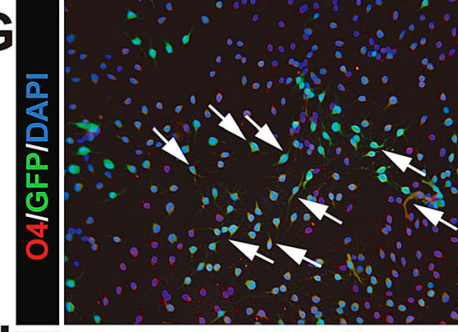

H

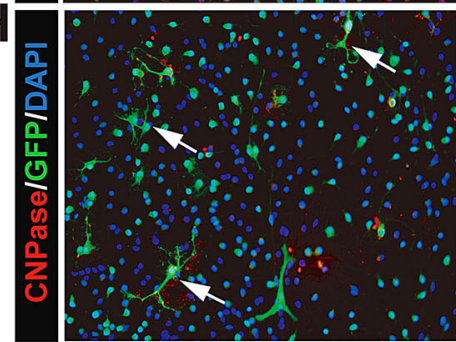

I

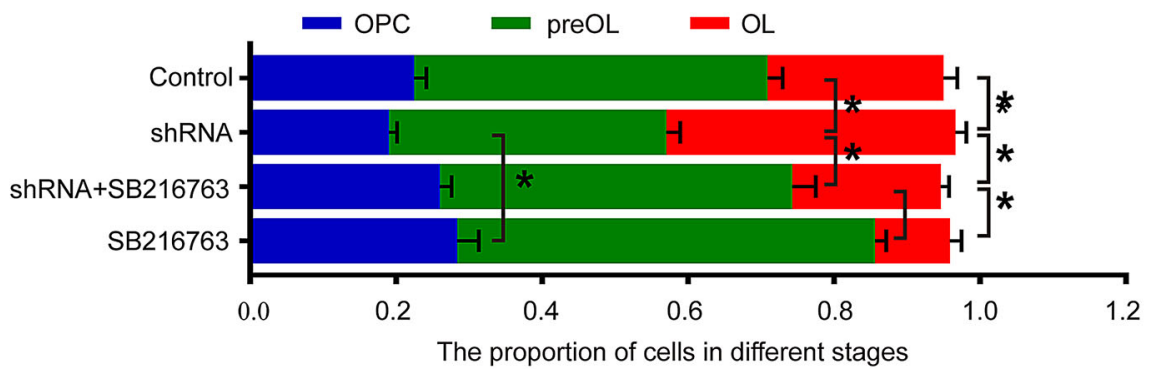

Springer

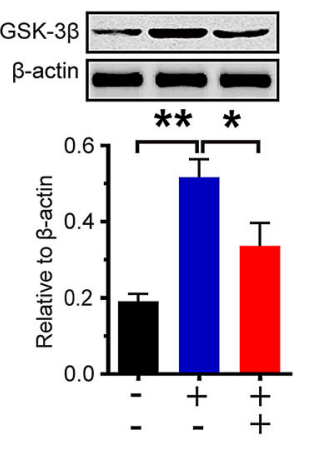

B
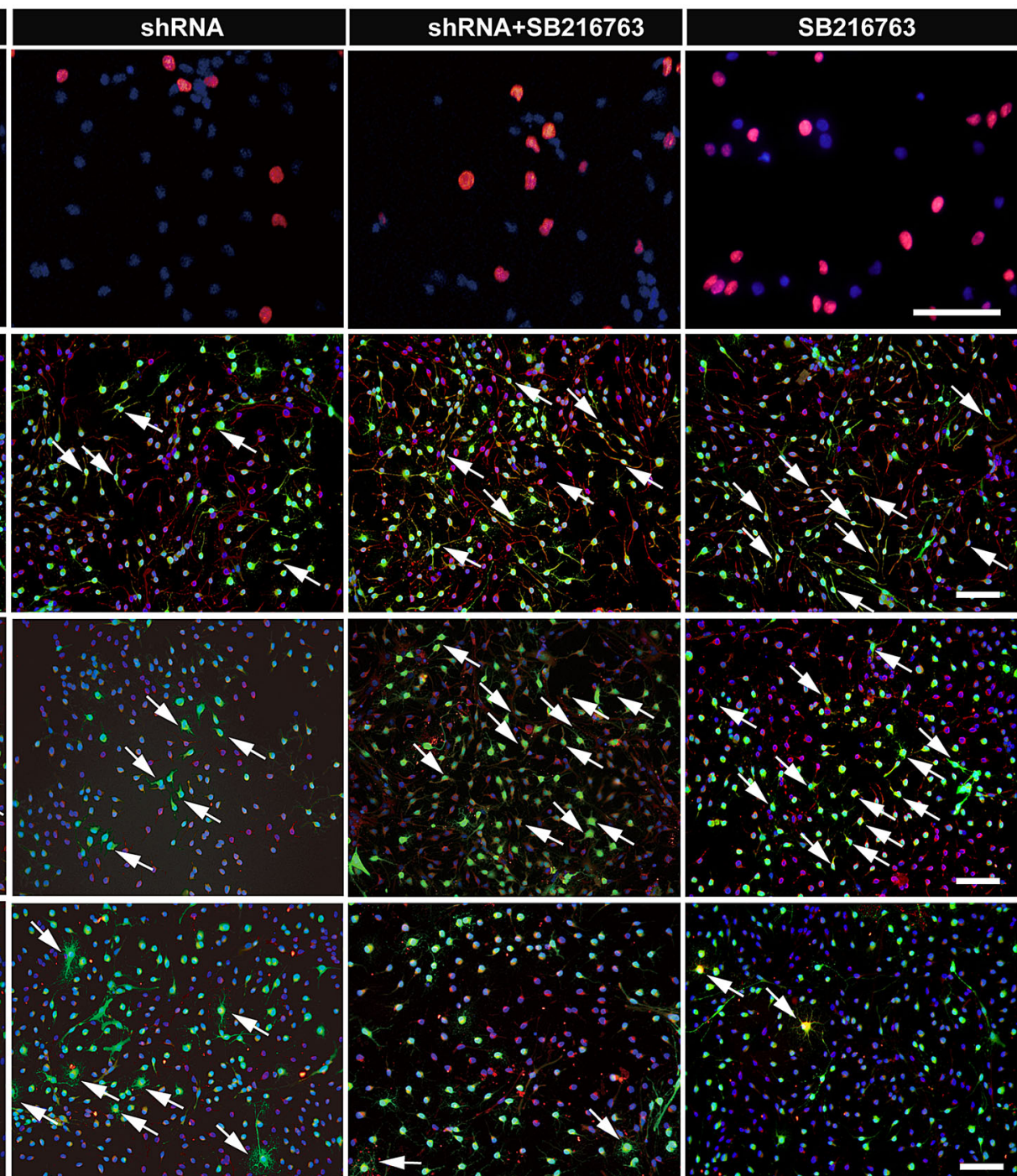

$\therefore$
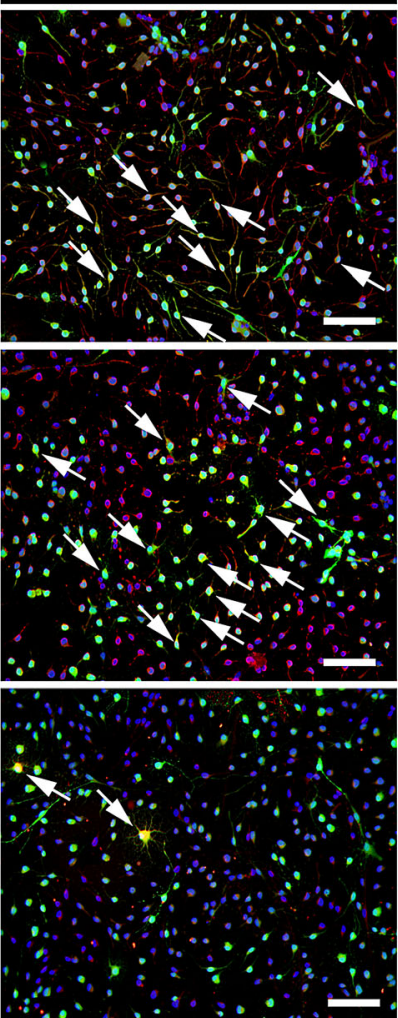

E

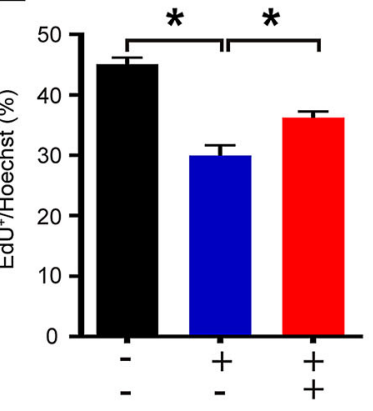

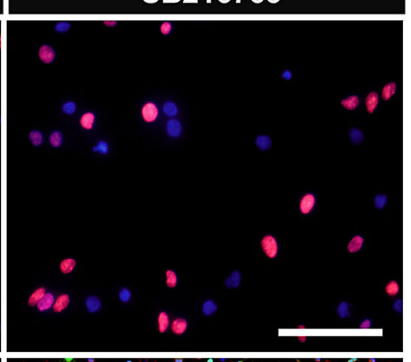


4Fig. 7 Foxg1 regulates the proliferation and differentiation of OPCs by the Wnt signaling pathway. A GSK-3 $\beta$ mRNA expression in each group of cells assessed by qRT-PCR. B, C Western blots and quantitative analyses of the protein expression of GSK-3 $\beta$ (B) and nuclear $\beta$-catenin $(\mathbf{C})$ in each group of cells. D, E EdU staining (D) and quantitative analysis (E) in each group of cells. F, G Representative images of NG2 (F) and $\mathrm{O} 4$ (G) immunofluorescence staining in each group of cells. $\mathbf{H}$ Representative images of CNPase immunofluorescence staining in each group of cells cultured in DMEM/F12 medium containing 10\% FBS without PDGF-AA and bFGF for 7 days. I Cell proportions at different stages during the differentiation of OPCs. White arrows, representative double-labeled positive cells; $n=6$ for each group; data are presented as the mean \pm SEM; $* P<0.05, * * P<0.01$, one-way ANOVA with Tukey's post hoc test; scale bars, $40 \mu \mathrm{m}$.

\section{Discussion}

As described in previous reports, Foxgl is abundantly expressed in the CNS [31] and plays pivotal roles in organogenesis through the regulation of proliferation and the specification of cell fate [32]. However, the role of Foxg1 in the oligodendroglial lineage and the underlying mechanisms remain unclear. Therefore, the present study was designed to determine the effect of Foxgl and the mechanisms underlying the regulation of the Wnt signaling pathway following CPZ-induced demyelination.

$\mathrm{CPZ}$ is a copper chelator that impacts cell metabolism and leads to oligodendrocyte death and demyelination accompanied by weight loss and behavioral disorders [33]. The CPZ-fed C57BL/6 mouse model has been increasingly used to study demyelination and remyelination in the CNS $[33,34]$. The present results are consistent with previous reports [35, 36], demonstrating that we successfully replicated the CPZ-induced demyelination model in the ICR strain of mice [37]. However, for the first time, we found that conditional knockout of Foxgl in NSPCs reversed the demyelination and spatial learning and memory impairments; moreover, a more evident remyelination was found after CPZ withdrawal. It has been reported that different subcellular localizations of Foxg1 control the machinery that causes cell differentiation, replication, and bioenergetics. A fraction of Foxgl is recruited to mitochondria and plays a major role in neuronal survival, differentiation, and plasticity [38]. In addition, dietary triheptanoin rescues oligodendrocyte loss in a mouse model of Canavan disease [39], as well as the functional and molecular abnormalities in $\mathrm{Foxgl}^{+/-}$mice [40]. Thus, it should be noted that the food used in our study was a normal diet. To induce demyelination, $0.2 \%$ (w/w) CPZ was mixed into ground standard rodent chow. In addition, each group of mice received the same amount of chow. Thus, we confirmed that Foxgl might participate in the repair of myelin sheath damage in mice with CPZinduced demyelination.
Oligodendrocytes are derived from OPCs [41], which populate the CNS starting from embryonic development [42, 43]. Previous studies [44, 45] have demonstrated that demyelinating injury induces OPC proliferation; however, studies on the differentiation of OPCs into mature oligodendrocytes and remyelination are limited. The mechanisms underlying the effect of Foxgl on the regulation of demyelination and remyelination remain to be clarified. Here, we found that $\mathrm{CPZ}$ administration increased the number of OPCs, which indicated that CPZ injury initiates the endogenous repair process and promotes the production of OPCs from neural stem cells of the subventricular zone region and migration to the injured area [46]. In the CPZinduced demyelinated mouse corpus callosum, OPC proliferation was decreased by Foxg 1 cKO. Moreover, double labeling using BrdU and cell-specific markers showed that Foxgl cKO promoted OPC differentiation into mature OLs. Previous research has demonstrated that Foxg1 may be necessary for maintenance of the NSC pool in the CNS and that genetic inactivity of Foxgl promotes both gliogenesis and neurogenesis [11]. NSCs are maintained in a quiescent state in the adult CNS [47]. Although it has been reported that Foxgl cKO in nestin-positive NSCs promotes neurogenesis and gliogenesis [11], it did not markedly change the myelin sheath and behavior in mice fed a normal diet in our study. We conclude that the change was so mild that there was no marked qualitative difference between the groups. However, the CPZ diet induced very serious demyelination accompanied by a change in the stem cell niche and OPC proliferation, and under this pathological situation, Foxgl cKO clearly promoted OPC differentiation. In vitro, purified primary OPCs were used to assess the effects of Foxgl inhibition on OPC proliferation and differentiation. It has been demonstrated that the loss of Foxgl leads to cycle exit and promotes the differentiation of premature cells [48, 49]. Consistent with previous reports [9-11], Foxgl knockdown inhibited the proliferation and promoted the differentiation of OPCs under normal culture conditions. As a result, the number of OPCs in the CPZ-treated corpus callosum area was reduced due to accelerated differentiation. The differentiated mature OLs encapsulated the axons of the neurons to form new myelin sheaths, thereby alleviating the myelin damage caused by CPZ or accelerating remyelination.

The $\mathrm{Wnt} / \beta$-catenin signaling pathway plays an important role in regulating cell proliferation and differentiation [50-52]. Studies have shown that inhibition of GSK-3 $\beta$ expression in the Wnt signaling pathway inhibits the differentiation of OPCs while promoting their proliferation $[53,54]$. Here, we found a significant increase in GSK-3 $\beta$ at the mRNA and protein levels in OPCs after Foxgl knockdown. We used $10 \mu \mathrm{mol} / \mathrm{L}$ SB216763 to inhibit the function of GSK-3 $\beta[55,56]$, and found that this treatment 
reversed the effect of Foxgl on OPC proliferation and differentiation and the nuclear level of $\beta$-catenin. These results are in agreement with reports in the literature [15]. Together with the results of the in vivo experiments performed here, it can be concluded that conditional knockout or knockdown of Foxgl effectively facilitates the differentiation of OPCs into OLs and promote the recovery of damaged myelin, and the $\mathrm{Wnt} / \beta$-catenin signaling pathway is involved in this process. Given the increasing importance of glia-glia crosstalk and the inflammatory microenvironment in demyelination or remyelination [57-59], future studies are needed to focus on the microglia, astrocytes, and even the interaction between microglia, astrocytes, and oligodendrocytes, to further clarify the role of the $\mathrm{Wnt} / \beta$-catenin signaling pathway in CPZ-induced demyelination or remyelination.

In summary, the present study provides evidence that, in CPZ-induced demyelinating mice, Foxgl knockout inhibits the proliferation but promotes the differentiation of OPCs into mature OLs by regulating the activity of the Wnt $/ \beta$ catenin signaling pathway, which ultimately reduces the severity of demyelination and promotes remyelination. Therefore, Foxgl might be a promising novel target to enhance endogenous remyelination in patients with demyelinating diseases such as MS. Further experiments are required to investigate the precise targets of Foxgl in the Wnt/ $\beta$-catenin signaling pathway and its interactions with other types of glial cells involved in demyelinating diseases in the CNS.

Acknowledgements We sincerely thank Professor Chunjie Zhao from the Medical School of Southeast University for providing the Foxg1 cKO mice. This work was supported by the National Natural Science Foundation of China (81771337 and 81271345), the National Key R\&D Program of China (2017YFA0104202), the Natural Science Foundation of Jiangsu Province (BK20161174), the Natural Science Foundation of the Jiangsu Higher Education Institutions of China (18KJB180028), and the Six Talent Peaks Project in Jiangsu Province (2015 to RY).

Conflict of interest The authors have no conflicts of interest to declare.

Open Access This article is licensed under a Creative Commons Attribution 4.0 International License, which permits use, sharing, adaptation, distribution and reproduction in any medium or format, as long as you give appropriate credit to the original author(s) and the source, provide a link to the Creative Commons licence, and indicate if changes were made. The images or other third party material in this article are included in the article's Creative Commons licence, unless indicated otherwise in a credit line to the material. If material is not included in the article's Creative Commons licence and your intended use is not permitted by statutory regulation or exceeds the permitted use, you will need to obtain permission directly from the copyright holder. To view a copy of this licence, visit http://creativecommons. org/licenses/by/4.0/.

\section{References}

1. Lu F, Yin D, Pu Y, Liu W, Li Z, Shao Q, et al. Shikimic acid promotes oligodendrocyte precursor cell differentiation and accelerates remyelination in mice. Neurosci Bull 2019, 35: 434-446.

2. Fan HB, Chen LX, Qu XB, Ren CL, Wu XX, Dong FX, et al. Transplanted miR-219-overexpressing oligodendrocyte precursor cells promoted remyelination and improved functional recovery in a chronic demyelinated model. Sci Rep 2017, 7: 41407.

3. Amaral AI, Tavares JM, Sonnewald U, Kotter MR. Oligodendrocytes: Development, physiology and glucose metabolism. Adv Neurobiol 2016, 13: 275-294.

4. Pan Y, Jiang Z, Sun D, Li Z, Pu Y, Wang D, et al. Cyclindependent kinase 18 promotes oligodendrocyte precursor cell differentiation through activating the extracellular signal-regulated kinase signaling pathway. Neurosci Bull 2019, 35: 802-814.

5. Murphy DB, Wiese S, Burfeind P, Schmundt D, Mattei MG, Schulz-Schaeffer W, et al. Human brain factor 1, a new member of the fork head gene family. Genomics 1994, 21: 551-557.

6. Eagleson KL, Schlueter McFadyen-Ketchum LJ, Ahrens ET, Mills PH, Does MD, Nickols J, et al. Disruption of Foxg1 expression by knock-in of cre recombinase: effects on the development of the mouse telencephalon. Neuroscience 2007, 148: 385-399.

7. Boggio EM, Pancrazi L, Gennaro M, Lo Rizzo C, Mari F, Meloni I, et al. Visual impairment in FOXG1-mutated individuals and mice. Neuroscience 2016, 324: 496-508.

8. Perche O, Haddad G, Menuet A, Callier P, Marcos M, Briault S, et al. Dysregulation of FOXG1 pathway in a 14q12 microdeletion case. Am J Med Genet A 2013, 161A: 3072-3077.

9. Martynoga B, Morrison H, Price DJ, Mason JO. Foxg1 is required for specification of ventral telencephalon and region-specific regulation of dorsal telencephalic precursor proliferation and apoptosis. Dev Biol 2020, 15: 107-121.

10. Brunetti-Pierri N, Paciorkowski AR, Ciccone R, Della Mina E, Bonaglia MC, Borgatti R, et al. Duplications of FOXG1 in 14q12 are associated with developmental epilepsy, mental retardation, and severe speech impairment. Eur J Hum Genet 2011, 19: 102-107.

11. Tian CX, Gong YF, Yang Y, Shen W, Wang K, Liu JH, et al. Foxg1 has an essential role in postnatal development of the dentate gyrus. J Neurosci 2012, 32: 2931-2949.

12. Gallo V, Deneen B. Glial development: the crossroads of regeneration and repair in the CNS. Neuron 2014, 83: 283-308.

13. Feigenson K, Reid M, See J, Crenshaw EB, Grinspan JB. Wnt signaling is sufficient to perturb oligodendrocyte maturation. Mol Cell Neurosci 2009, 42: 255-265.

14. Caspi M, Zilberberg A, Eldar-Finkelman H, Rosin-Arbesfeld R. Nuclear GSK-3beta inhibits the canonical Wnt signalling pathway in a beta-catenin phosphorylation-independent manner. Oncogene 2008, 27: 3546-3555.

15. Zhou L, Shao CY, Xu SM, Ma J, Xie YJ, Zhou L, et al. GSK3beta promotes the differentiation of oligodendrocyte precursor cells via beta-catenin-mediated transcriptional regulation. Mol Neurobiol 2014, 50: 507-519.

16. Han X, Gu X, Zhang Q, Wang Q, Cheng Y, Pleasure SJ, et al. FoxG1 directly represses dentate granule cell fate during forebrain development. Front Cell Neurosci 2018, 12: 452.

17. Li T, Wang J, Wang H, Yang Y, Wang S, Huang N, et al. The deletion of dicer in mature myelinating glial cells causes progressive axonal degeneration but not overt demyelination in adult mice. Glia 2018, 66: 1960-1971.

18. Liu M, Liu X, Wang L, Wang Y, Dong F, Wu J, et al. TRPV4 inhibition improved myelination and reduced glia reactivity and inflammation in a cuprizone-induced mouse model of demyelination. Front Cell Neurosci 2018, 12: 392. 
19. Liu S, Ren C, Qu X, Wu X, Dong F, Chand YK, et al. miR-219 attenuates demyelination in cuprizone-induced demyelinated mice by regulating monocarboxylate transporter 1 . Eur J Neurosci 2017, 45: 249-259.

20. Dong F, Wang S, Wang Y, Yang X, Jiang J, Wu D, et al. Quercetin ameliorates learning and memory via the Nrf2-ARE signaling pathway in d-galactose-induced neurotoxicity in mice. Biochem Biophys Res Commun 2017, 491: 636-641.

21. Qi DS, Tao JH, Zhang LQ, Li M, Wang M, Qu R, et al. Neuroprotection of Cilostazol against ischemia/reperfusion-induced cognitive deficits through inhibiting JNK3/caspase- 3 by enhancing Akt1. Brain Res 2016, 1653: 67-74.

22. Liu Y, Dong F, Guo R, Zhang Y, Qu X, Wu X, et al. Hydrogenrich saline ameliorates experimental autoimmune encephalomyelitis in C57BL/6 mice via the Nrf2-ARE signaling pathway. Inflammation 2019, 42: 586-597.

23. Dong F, Yao R, Yu H, Liu Y. Neuroprotection of Ro25-6981 Against ischemia/reperfusion-induced brain injury via inhibition of autophagy. Cell Mol Neurobiol 2017, 37: 743-752.

24. Lai Q, Du W, Wu J, Wang X, Li X, Qu X, et al. H3K9ac and HDAC2 activity are involved in the expression of monocarboxylate transporter 1 in oligodendrocyte. Front Mol Neurosci 2017, 10: 376.

25. Wang XQ, Yao RQ, Liu X, Huang JJ, Qi DS, Yang LH. Quercetin protects oligodendrocyte precursor cells from oxygen/ glucose deprivation injury in vitro via the activation of the PI3K/ Akt signaling pathway. Brain Res Bull 2011, 86: 277-284.

26. Li L, Chen HZ, Wang M, Chen FF, Gao J, Sun S, et al. NCAM140 Translocation into lipid rafts mediates the neuroprotective effects of GDNF. Mol Neurobiol 2017, 54: 2739-2751.

27. Bowler EH, Smith-Vidal A, Lester A, Bell J, Wang Z, Bell CG, et al. Deep proteomic analysis of Dnmt1 mutant/hypomorphic colorectal cancer cells reveals dysregulation of epithelial-mesenchymal transition and subcellular re-localization of BetaCatenin. Epigenetics 2020, 15: 107-121.

28. Qu X, Guo R, Zhang Z, Ma L, Wu X, Luo M, et al. bFGF protects pre-oligodendrocytes from oxygen/glucose deprivation injury to ameliorate demyelination. Cell Mol Neurobiol 2015, 35: 913-920.

29. Franco-Pons N, Torrente M, Colomina MT, Vilella E. Behavioral deficits in the cuprizone-induced murine model of demyelination/ remyelination. Toxicol Lett 2007, 169: 205-213.

30. Xiao L, Xu H, Zhang Y, Wei Z, He J, Jiang W, et al. Quetiapine facilitates oligodendrocyte development and prevents mice from myelin breakdown and behavioral changes. Mol Psychiatry 2008, 13: 697-708.

31. Pauley S, Lai E, Fritzsch B. Foxg1 is required for morphogenesis and histogenesis of the mammalian inner ear. Dev Dyn 2006, 235: 2470-2482.

32. Solomon KS, Logsdon JM, Jr., Fritz A. Expression and phylogenetic analyses of three zebrafish FoxI class genes. Dev Dyn 2003, 228: 301-307.

33. Torkildsen O, Brunborg LA, Myhr KM, Bo L. The cuprizone model for demyelination. Acta Neurol Scand Suppl 2008, 188: 72-76.

34. Zendedel A, Beyer C, Kipp M. Cuprizone-induced demyelination as a tool to study remyelination and axonal protection. J Mol Neurosci 2013, 51: 567-572.

35. Hoyos HC, Marder M, Ulrich R, Gudi V, Stangel M, Rabinovich GA, et al. The role of Galectin-3: From oligodendroglial differentiation and myelination to demyelination and remyelination processes in a cuprizone-induced demyelination model. Adv Exp Med Biol 2016, 949: 311-332.

36. Zhang Y, Yin L, Zheng N, Zhang L, Liu J, Liang W, et al. Icariin enhances remyelination process after acute demyelination induced by cuprizone exposure. Brain Res Bull 2017, 130: 180-187.

37. Pringproa K, Sathanawongs A, Khamphilai C, Sukkarinprom S, Oranratnachai A. Intravenous transplantation of mouse embryonic stem cells attenuates demyelination in an ICR outbred mouse model of demyelinating diseases. Neural Regen Res 2016, 11: 1603-1609.

38. Pancrazi L, Di Benedetto G, Colombaioni L, Della Sala G, Testa G, Olimpico F, et al. Foxg1 localizes to mitochondria and coordinates cell differentiation and bioenergetics. Proc Natl Acad Sci U S A 2015, 112: 13910-13915.

39. Francis JS, Markov V, Leone P. Dietary triheptanoin rescues oligodendrocyte loss, dysmyelination and motor function in the nur7 mouse model of Canavan disease. J Inherit Metab Dis 2014, 37: $369-381$

40. Testa G, Mainardi M, Olimpico F, Pancrazi L, Cattaneo A, Caleo $\mathrm{M}$, et al. A triheptanoin-supplemented diet rescues hippocampal hyperexcitability and seizure susceptibility in FoxG1 $(+/-)$ mice. Neuropharmacology 2019, 148: 305-310.

41. Simons M, Nave KA. Oligodendrocytes: Myelination and axonal support. Cold Spring Harb Perspect Biol 2015, 8: a020479.

42. Liu X, Lu Y, Zhang Y, Li Y, Zhou J, Yuan Y, et al. Slit2 regulates the dispersal of oligodendrocyte precursor cells via Fyn/ RhoA signaling. J Biol Chem 2012, 287: 17503-17516.

43. Kessaris N, Fogarty M, Iannarelli P, Grist M, Wegner M, Richardson WD. Competing waves of oligodendrocytes in the forebrain and postnatal elimination of an embryonic lineage. Nat Neurosci 2006, 9: 173-179.

44. Kremer D, Aktas O, Hartung HP, Kury P. The complex world of oligodendroglial differentiation inhibitors. Ann Neurol 2011, 69: 602-618.

45. Kuhlmann T, Miron V, Cui Q, Wegner C, Antel J, Bruck W. Differentiation block of oligodendroglial progenitor cells as a cause for remyelination failure in chronic multiple sclerosis. Brain 2008, 131: 1749-1758.

46. Emery B. Regulation of oligodendrocyte differentiation and myelination. Science 2010, 330: 779-782.

47. Sozmen EG, DiTullio DJ, Rosenzweig S, Hinman JD, Bridges SP, Marin MA, et al. White matter stroke induces a unique oligo-astrocyte niche that inhibits recovery. J Neurosci 2019, 39: 9343-9359.

48. Yu J, Li C, Ding Q, Que J, Liu K, Wang H, et al. Netrin-1 ameliorates blood-brain barrier impairment secondary to ischemic stroke via the activation of PI3K pathway. Front Neurosci 2017, 11: 700.

49. Bai L, Mei X, Shen Z, Bi Y, Yuan Y, Guo Z, et al. Netrin-1 improves functional recovery through autophagy regulation by activating the AMPK/mTOR signaling pathway in rats with spinal cord injury. Sci Rep 2017, 7: 42288.

50. Liu Z, Wu C, Xie N, Wang P. Long non-coding RNA MEG3 inhibits the proliferation and metastasis of oral squamous cell carcinoma by regulating the WNT/beta-catenin signaling pathway. Oncol Lett 2017, 14: 4053-4058.

51. Song H, Shi L, Xu Y, Xu T, Fan R, Cao M, et al. BRD4 promotes the stemness of gastric cancer cells via attenuating miR-216a-3pmediated inhibition of Wnt/beta-catenin signaling. Eur J Pharmacol 2019, 852: 189-197.

52. Zhang H, Qi Y, Geng D, Shi Y, Wang X, Yu R, et al. Expression profile and clinical significance of Wnt signaling in human gliomas. Oncol Lett 2018, 15: 610-617.

53. Ye P, Hu Q, Liu H, Yan Y, D'Ercole A J. beta-catenin mediates insulin-like growth factor-I actions to promote cyclin D1 mRNA expression, cell proliferation and survival in oligodendroglial cultures. Glia 2010, 58: 1031-1041.

54. Azim K, Butt AM. GSK3beta negatively regulates oligodendrocyte differentiation and myelination in vivo. Glia 2011, 59: 540-553.

55. Heyd F, Lynch KW. Phosphorylation-dependent regulation of PSF by GSK3 controls CD45 alternative splicing. Mol Cell 2010, 40: $126-137$.

56. Martin M, Rehani K, Jope RS, Michalek SM. Toll-like receptormediated cytokine production is differentially regulated by glycogen synthase kinase 3. Nature Immunology 2005, 6: 777-784. 
57. Tepavcevic V, Kerninon C, Aigrot MS, Meppiel E, Mozafari S, Arnould-Laurent R, et al. Early netrin-1 expression impairs central nervous system remyelination. Ann Neurol 2014, 76: 252-268.

58. Rajasekharan S, Bin JM, Antel JP, Kennedy TE. A central role for RhoA during oligodendroglial maturation in the switch from netrin-1-mediated chemorepulsion to process elaboration. J Neurochem 2010, 113: 1589-1597.

59. He X, Li Y, Lu H, Zhang Z, Wang Y, Yang GY. Netrin-1 overexpression promotes white matter repairing and remodeling after focal cerebral ischemia in mice. J Cereb Blood Flow Metab 2013, 33: 1921-1927. 